\title{
Exchangeable measures for subshifts
}

\author{
J. Aaronson ${ }^{\mathrm{a}, 1}, \mathrm{H}$. Nakada ${ }^{\mathrm{b}, 2}$, O. Sarig ${ }^{\mathrm{c}, 3, *}$ \\ a School of Math. Sciences, Tel Aviv University, 69978 Tel Aviv, Israel \\ ${ }^{\mathrm{b}}$ Dept. of Math., Keio University, Hiyoshi 3-14-1 Kohoku, Yokohama 223, Japan \\ ${ }^{c}$ Dept. of Math., Penn State University, University Park, PA 16802, USA
}

Received 15 September 2004; received in revised form 29 August 2005; accepted 13 October 2005

Available online 11 July 2006

\begin{abstract}
Let $\Omega$ be a Borel subset of $S^{\mathbb{N}}$ where $S$ is countable. A measure is called exchangeable on $\Omega$, if it is supported on $\Omega$ and is invariant under every Borel automorphism of $\Omega$ which permutes at most finitely many coordinates. De-Finetti's theorem characterizes these measures when $\Omega=S^{\mathbb{N}}$. We apply the ergodic theory of equivalence relations to study the case $\Omega \neq S^{\mathbb{N}}$, and obtain versions of this theorem when $\Omega$ is a countable state Markov shift, and when $\Omega$ is the collection of beta expansions of real numbers in $[0,1]$ (a non-Markovian constraint).

(C) 2006 Elsevier Masson SAS. All rights reserved.

\section{Résumé}

Soit $\Omega$ un sous-ensemble borélien de $S^{\mathbb{N}}$ où $S$ est dénombrable. Une mesure est dite échangeable sur $\Omega$, si elle est portée par $\Omega$ et est invariante par chaque automorphisme borélien de $\Omega$ qui permute au plus un nombre fini de coordonnées. Le théorème de DeFinetti caractérise ces mesures quand $\Omega=S^{\mathbb{N}}$. Nous appliquons la théorie ergodique des relations d'équivalence pour étudier le cas $\Omega \neq S^{\mathbb{N}}$, et obtenons des versions de ce théorème quand $\Omega$ est une chaîne de Markov topologique d'un espace d'états dénombrable, et quand $\Omega$ est la collection des "beta-développement" des nombres réels dans [0,1] (une contrainte non-markovienne).

(c) 2006 Elsevier Masson SAS. All rights reserved.
\end{abstract}

MSC: 60G09; 37A20

Keywords: Exchangeability; Tail equivalence relations; Beta expansions; Countable Markov shifts

\section{Introduction}

Exchangeability. De-Finetti's theorem says that if a stochastic process $\left\{X_{n}\right\}_{n} \geqslant 1$ is exchangeable, i.e. all finite permutations $\left\{X_{\pi(n)}\right\}$ of $\left\{X_{n}\right\}_{n} \geqslant 1$ are distributed like $\left\{X_{n}\right\}$, then it is distributed as a mixture of i.i.d. distributions.

\footnotetext{
* Corresponding author.

E-mail addresses: aaro@tau.ac.il (J. Aaronson), nakada@math.keio.ac.jp (H. Nakada), sarig@ math.psu.edu (O. Sarig).

1 Tel.: +972 3 6408805; fax: +97236409357.

2 Tel.: +8145566 1641; fax: +81455661642.

3 The third author acknowledges support of NSF grant DMS-0500630. Tel.: +1 814 8639678; fax: +1 8148653735 .
} 
Here is a seemingly stronger, but equivalent formulation: Let $\mathfrak{K}$ be the collection of all bi-measurable bijections $\kappa: A \rightarrow B\left(A, B \subseteq S^{\mathbb{N}}\right.$ Borel) for which for every $x \kappa(x)$ is some finite permutation ${ }^{4}$ of $x$; then any Borel probability measure $m$ on $\Omega:=S^{\mathbb{N}}$ such that $\left.m \circ \kappa\right|_{\operatorname{Dom}(\kappa)}=\left.m\right|_{\operatorname{Dom}(\kappa)}$ for all $\kappa \in \mathfrak{K}$ is an average of Bernoulli measures.

De-Finetti's theorem is instrumental in statistical modeling of sequential sampling, because it determines the form of joint distributions whenever the sampling order is unimportant. But sometimes the sampling order is subject to non-permutation invariant deterministic constraints. In these cases the joint distribution cannot be assumed to be exchangeable. Nevertheless, one can still ask for 'the most exchangeable' compatible distributions.

There are various ways to formalize this. In this paper we use the following: Let $\Omega$ be a Borel subset of $S^{\mathbb{N}}$ (thought of as the space of realizations of $\left\{X_{n}\right\}_{n} \geqslant 1$ subject to a collection of deterministic constraints), and set $\mathfrak{K}(\Omega):=\{\kappa \in \mathfrak{K}$ : $\operatorname{Dom}(\kappa), \operatorname{Im}(\kappa) \subseteq \Omega\}$. A Borel measure $m$ on $\Omega$ is called exchangeable on $\Omega$ if $\left.m \circ \kappa\right|_{\operatorname{Dom}(\kappa)}=\left.m\right|_{\operatorname{Dom}(\kappa)}$ for all $\kappa \in \mathfrak{K}(\Omega)$. When $\Omega=S^{\mathbb{N}}$, this reduces to the usual notion of exchangeability.

This definition of exchangeability is the one used by Petersen and Schmidt in the context of finite state Markov shifts [23], but is not equivalent to the definition of 'partial exchangeability' introduced by Diaconis and Freedman in the context of topological Markov shifts [8]. (Topological Markov shifts are sample spaces of Markov chains, see Section 3 below.)

The shift invariant exchangeable measures for two sided finite state topological Markov shifts were determined by Petersen and Schmidt [23]. The exchangeable measures for a one sided finite state topological Markov shift were determined by Aaronson, Nakada, Solomyak and Sarig in [5]. The partially exchangeable measures for countable state Markov topological shifts were determined by Diaconis and Freedman [8].

Aim. This paper describes exchangeable measures on $\Omega \subset S^{\mathbb{N}},|S| \leqslant \aleph_{0}$, in the following cases:

(1) Markov constraints: $\Omega$ is a one-sided countable state topological Markov shift (i.e. the sample space of a countable state Markov chain, see Section 3);

(2) A Non-Markov constraint: $\Omega$ is a $\beta$-shift (i.e. the collection of all (greedy) $\beta$-expansions of real $\theta \in[0,1]$, where $\beta>1$, see Section 7). The motivation for studying $\beta$-shifts comes from number theory (see $[24,21,27]$ and references therein).

Our results apply to probability measures as well as to locally finite infinite measures (see below). Such measures appear naturally in our context, because the state space $S$ is infinite.

We give a brief outline of our approach.

Equivalence relations. The sets $\Omega$ considered above are shift invariant: $T(\Omega)=\Omega$, where $T$ is the left shift map $T\left(x_{1}, x_{2}, \ldots\right)=\left(x_{2}, x_{3}, \ldots\right)$. We will use the language of equivalence relations reviewed below to formulate the exchangeability property in terms of some natural equivalence relations associated with certain skew-products over $T$ (see $[4,23]$ and below). This will allow us to bring in some tools from ergodic theory and thus bypass some of the combinatorial complications a direct approach would have encountered.

Let $(X, \mathcal{B}(X))$ be a standard measurable space. An equivalence relation on $X$ is a set $\mathcal{R} \subseteq X \times X$ such that the relation $x \sim y \Leftrightarrow(x, y) \in \mathcal{R}$ is an equivalence relation. An equivalence relation is called Borel if $\mathcal{R} \in \mathcal{B}(X) \otimes \mathcal{B}(X)$, and is called countable if all its equivalence classes $\mathcal{R}_{x}:=\{y:(y, x) \in \mathcal{R}\}(x \in X)$ are countable. Take as an example a countable discrete set $S$, a Borel set $\Omega \subseteq S^{\mathbb{N}}$ with the relative product topology, and $\mathcal{B}(\Omega)$ the Borel $\sigma$-algebra. The following set is a countable Borel equivalence relation on $\Omega$ :

$$
\mathcal{E}(\Omega):=\{(x, y) \in \Omega \times \Omega: x, y \text { differ by a finite permutation }\} .
$$

We later refer to $\mathcal{E}(\Omega)$ as the exchangeable relation of $\Omega$.

A bi-measurable bijection $\kappa$ defined on some $A \in \mathcal{B}$ with image $B \in \mathcal{B}$ is an $\mathcal{R}$-holonomy if $(x, \kappa(x)) \in \mathcal{R}$ for any $x \in A$. We write in this case $A \stackrel{\mathcal{R}}{\rightarrow} B$.

A function $F: X \rightarrow \mathbb{R}$ is called $\mathcal{R}$-invariant, if it is invariant under all $\mathcal{R}$-holonomies. A measure $m$ is called $\mathcal{R}$-ergodic, if all measurable $\mathcal{R}$-invariant functions are equal a.e. to a constant. Every $\mathcal{R}$-invariant measure can be decomposed into ergodic components, see Section 1 for details.

\footnotetext{
4 A permutation $\pi$ is called finite if its support $\{s: \pi(s) \neq s\}$ is finite.
} 
A measure on $(X, \mathcal{B}(X))$ is called $\mathcal{R}$-invariant, if $\left.m \circ \kappa\right|_{\operatorname{Dom}(\kappa)}=\left.m\right|_{\operatorname{Dom}(\kappa)}$ for all $\mathcal{R}$-holonomies $\kappa$ (with domain $\operatorname{Dom}(\kappa)$ ). The collection of $\mathcal{E}(\Omega)$-invariant measures is exactly the collection of exchangeable measures on $\Omega$.

Exchangeability, skew-products, and tail relations. We represent the exchangeable relation in terms of $T: \Omega \rightarrow \Omega$. Fix $a_{0} \in S$, consider the (additive) Abelian group

$$
\mathbb{Z}_{0}^{S \backslash\left\{a_{0}\right\}}:=\left\{x \in \mathbb{Z}^{S \backslash\left\{a_{0}\right\}} \text { : all but finitely many coordinates of } x \text { are zero }\right\}
$$

equipped with the discrete topology, and define $F^{\natural}: \Omega \rightarrow \mathbb{Z}_{0}^{\alpha \backslash\left\{a_{0}\right\}}$ by

$$
F^{\natural}\left(x_{0}, x_{1}, \ldots\right)_{a}:=\delta_{a, x_{0}} .
$$

Let $F_{k}^{\natural}:=F^{\natural}+F^{\natural} \circ T+\cdots+F^{\natural} \circ T^{k-1}$. These count the appearances of elements of $S$ in the first $k$ symbols of $x$. It is routine to verify that the exchangeable relation is the same as

$$
\mathfrak{T}\left(T, F^{\natural}\right):=\left\{(x, y) \in \Omega \times \Omega: \exists k \geqslant 0 \text { s.t. } T^{k} x=T^{k} y \text { and } F_{k}^{\natural}(x)=F_{k}^{\natural}(y)\right\} \text {. }
$$

It follows that exchangeability is the same as $\mathfrak{T}\left(T, F^{\natural}\right)$-invariance.

In order to study $\mathfrak{T}\left(T, F^{\natural}\right)$, we represent it in terms of the tail relation of a suitable transformation. This is done using standard abstract ergodic theoretic constructions which we now review. Let $X$ be a standard space.

(1) Tail relations: Let $T: X \rightarrow X$ be a measurable, locally invertible transformation on $X$. The tail relation of $T$ is

$$
\mathfrak{T}(T):=\left\{(x, y) \in X \times X: \exists k \geqslant 0 \text { s.t. } T^{k} x=T^{k} y\right\} .
$$

Define $X_{0}:=\{x \in X: x$ is not eventually periodic $\}$. The grand tail relation of $T$ is the equivalence relation

$$
\mathfrak{G}(T):=\left\{(x, y) \in X_{0} \times X_{0}: \exists k, \ell \geqslant 0 \text { s.t. } T^{k} x=T^{\ell} y\right\} .
$$

(2) Orbit cocycles: Let $\mathcal{R}$ be a countable Borel equivalence relation on $X$ and $\mathbb{G}$ an Abelian topological group. A Borel function $\Psi: \mathcal{R} \rightarrow \mathbb{G}$ is called an $\mathcal{R}$-cocycle if

$$
\Psi(x, z)=\Psi(x, y)+\Psi(y, z) \quad \text { whenever }(x, y),(y, z) \in \mathcal{R} .
$$

Any $F: X \rightarrow \mathbb{G}$ gives rise to the following $\mathfrak{T}(T)$ and $\mathfrak{G}(T)$-cocycles (which abusing notation we denote by the same symbol):

$$
\begin{array}{ll}
\widehat{F}(x, y):=F_{k}(y)-F_{k}(x), & (x, y) \in \mathfrak{T}(T) \text { and } T^{k} x=T^{k} y, \\
\widehat{F}(x, y):=F_{k}(y)-F_{\ell}(x), & (x, y) \in \mathfrak{G}(T) \text { and } T^{\ell} x=T^{k} y,
\end{array}
$$

where $F_{k}:=F+F \circ T+\cdots+F \circ T^{k-1}$.

(3) Skew-products: The skew-product relation with base $\mathcal{R}$ and cocycle $\Psi$ is the following countable equivalence relation on $X \times \mathbb{G}$ :

$$
\mathcal{R}_{\Psi}:=\left\{((x, t),(y, s)) \in(X \times \mathbb{G})^{2}:(x, y) \in \mathcal{R} \text { and } t-s=\Psi(x, y)\right\} .
$$

Note that $\mathfrak{T}(T)_{\widehat{F}} \equiv \mathfrak{T}\left(T_{F}\right)$, where $T_{F}: X \times \mathbb{G} \rightarrow X \times \mathbb{G}$ is the skew-product transformation $T_{F}(x, \xi):=(T x, \xi+$ $F(x))$.

(4) Inducing: Let $\mathcal{R}$ be as above and $E \subset X$ be some Borel set. The induced relation on $E$ is $\mathcal{R}(E):=\mathcal{R} \cap(E \times E)$. This is a countable Borel equivalence relation on $E$.

We now have the following identity:

$$
\mathcal{E}(\Omega)=\mathfrak{T}\left(T, F^{\natural}\right) \cong \mathfrak{T}(T) \widehat{F}^{\natural} \cap[\Omega \times\{0\}]^{2}=\mathfrak{T}\left(T_{F^{\natural}}\right)\left([\Omega \times\{0\}]^{2}\right),
$$

where the isomorphism $\cong$ is $(x, y) \leftrightarrow((x, 0),(y, 0))$.

It is a standard fact that if $\mathcal{R}$ is a countable Borel equivalence relation on $X$ and $E \subseteq X$ is Borel, then any $\mathcal{R}$-ergodic invariant measure restricts to an $\mathcal{R}(E)$-invariant ergodic measure on $E$, and that any $\mathcal{R}(E)$-ergodic invariant measure arises this way (see Proposition 1.0 below). 
This fact, together with identity (0.2) reduces the study of exchangeable measures on $\Omega$ to the study of $\mathfrak{T}\left(T_{F^{\natural}}\right) \equiv$ $\left(\mathfrak{T}(T) \widehat{F}_{F^{\natural}}\right.$-invariant measures on $\Omega \times \mathbb{Z}^{S \backslash\left\{a_{0}\right\}}$.

Conformal measures and the Maharam construction. The previous discussion shows that the exchangeability problem can be reduced to the study of invariant measures for the skew-product relation $\mathcal{R}_{\Psi}$, with $\mathcal{R}=\mathfrak{T}(T)$ and $\Psi=\widehat{F^{\natural}}$. There is a standard construction, called the Maharam construction after [19], of such measures.

Let $\mathcal{R}$ be a countable Borel equivalence relation on $X, \mathbb{G}$ a locally compact polish Abelian group (e.g. $\mathbb{Z}^{S \backslash\left\{a_{0}\right\}}$ ), $\Psi: \mathcal{R} \rightarrow \mathbb{G}$ an orbit cocycle, and $H: \mathbb{G} \rightarrow \mathbb{R}$ a continuous homomorphism. A measure $\mu$ on $X$ is $\mathcal{R}$-non-singular, if every $\mathcal{R}$-holonomy $\kappa: A \rightarrow B$ is non-singular, i.e., $\left.\left.m \circ \kappa\right|_{A} \sim m\right|_{A}$. An $\mathcal{R}$-non-singular measure $\mu$ is called $\left(\mathrm{e}^{H \circ \Psi}, \mathcal{R}\right)$ conformal if $\frac{\mathrm{d} \mu \circ \kappa}{\mathrm{d} \mu}(x)=\mathrm{e}^{H \circ \Psi(x, \kappa x)}$ a.e. on Dom $\kappa$ for all holonomies $\kappa$.

The Maharam measure corresponding to a $\left(\mathrm{e}^{H \circ \Psi}, \mathcal{R}\right)$-conformal measure $\mu$ on $X$ is the following measure on $X \times \mathbb{G}:$

$$
\mathrm{d} m(x, y):=\mathrm{e}^{-H(y)} \mathrm{d} \mu(x) \mathrm{d} m_{\mathbb{G}}(y),
$$

where $m_{\mathbb{G}}$ is a Haar measure for $\mathbb{G}$. It is straightforward to check that such measures are $\mathcal{R}_{\Psi}$-invariant. Ergodicity is not guaranteed even when $\mu$ is $\mathcal{R}$-ergodic.

It is instructive to interpret the Maharam measures in the special case $X=\Omega, \mathbb{G}=\mathbb{Z}^{S \backslash\left\{a_{0}\right\}}, \mathcal{R}=\mathfrak{T}(T)$, and $\Psi=\widehat{F^{\natural}}$. In this case any Maharam measure on $\mathfrak{T}(T)_{\widehat{F}}$ restricts to the measure $\mu \times \delta_{0}$ on $\mathfrak{T}(T) \widehat{F^{\natural}} \cap(\Omega \times\{0\})^{2}$. The isomorphism $\cong$ carries this measure to $\mu$, and identity (0.2) shows that this measure must be exchangeable.

Thus every $\left(\mathrm{e}^{H \circ F^{\natural}}, \mathfrak{T}(T)\right)$-conformal measure is exchangeable. In this paper we study the other direction: starting with an arbitrary exchangeable measure, we ask to what extent can it be constructed from conformal measures. We do this for two particular choices of $\Omega$ : countable Markov shifts, and sets of $\beta$-expansions.

Programme. For the sets $\Omega$ described above we shall do the following:

(1) Establish the existence of conformal measures, and identify them;

(2) Characterize their $\mathfrak{T}\left(T, F^{\natural}\right)$-ergodicity;

(3) Show, or find sufficient conditions for $\mathfrak{T}\left(T, F^{\natural}\right)$-invariant ergodic measures to be ergodic conformal measures when restricted to their support.

We compare the Markovian and non-Markovian situations. The study of conformal measures in the non-Markovian case ( $\beta$-expansions) requires different tools than in the Markovian case (countable Markov shifts). There is however a common thread in part (3).

Rather than showing that $\mathfrak{T}\left(T, F^{\natural}\right)$-ergodic invariant locally finite measures are conformal, we show that $\mathfrak{T}(T) \widehat{F}^{\natural}$ ergodic invariant locally finite measures $m$ are Maharam. The key step is to show quasi-invariance under all transformations of the form $Q_{a}(x, \xi):=(x, \xi+a)$, as this implies the Maharam form for abstract reasons (see the proof of Theorem 5.0 or 9.0). To do this we construct explicit approximations to $Q_{a}$ by $\mathcal{R}_{\Psi}$-holonomies.

The construction of the approximating holonomies depends, of course, on the structure of the $\Omega$ in question.

Various parts of this programme make sense for more general cocycles than $F^{\natural}$, and a larger class of subshifts than those considered here. It is of some interest to identify particular properties which are sufficient for our argument to work. We therefore carry out parts of this programme in greater generality than needed for the exchangeability problem.

The paper is divided into three parts. In the first, we collect some terminology, notation, and facts from the ergodic theory of equivalence relations. We also solve the exchangeability problem for locally finite infinite measures on fullshifts. In the second and third parts we treat, respectively, exchangeable measures for countable Markov shifts, and for $\beta$-expansions.

\section{Part I: Generalities}

\section{More on equivalence relations and conformal measures}

Some notions of finiteness for measures. Let $(X, \mathcal{B}(X))$ be a standard measurable space. The collections of probabilities, and $\sigma$-finite measures on a standard measurable space $X$ are denoted by $\mathcal{P}(X)$ and $\mathfrak{M}(X)$ respectively. 
If $\alpha \subset \mathcal{B}(X)$ is a countable partition, we set $\mathfrak{M}_{\alpha}(X):=\{\mu \in \mathfrak{M}(X): \mu(A)<\infty \forall A \in \alpha\}$ and call these measures $\alpha-\sigma$-finite.

If $X$ is equipped with a topology (generating its measurable structure), we call $\mu \in \mathfrak{M}(X)$ locally finite (on $X$ ) if there is a countable cover of $X$ by open sets, each with finite $\mu$-measure, and topologically $\sigma$-finite if it is locally finite on some Borel subset of full $\mu$-measure.

The Feldman-Moore Theorem. The definition of ergodicity and invariance under an equivalence relation includes quantification over all holonomies. This is unnecessary.

A collection $\mathfrak{C}$ of $\mathcal{R}$-holonomies generates $\mathcal{R}$ if for each $(x, y) \in \mathcal{R}, \exists \Phi \in \mathfrak{C}$ such that $x \in \operatorname{Dom} \Phi, y=\Phi(x)$. A measure is $\mathcal{R}$-invariant iff it is invariant w.r.t. to a generating collection of holonomies. A function is $\mathcal{R}$-invariant iff it is invariant w.r.t. a generating collection of holonomies.

Feldman and Moore proved in [10] that any countable Borel equivalence relation is generated by a countable group of globally defined holonomies: $\exists \Gamma$ a group of Borel automorphisms s.t. $\mathcal{R}=\mathcal{R}_{\Gamma}:=\{(x, g(x)): x \in X, g \in \Gamma\}$.

The ergodic decomposition. For a countable Borel equivalence relation, a set $A \subset X$ is $\mathcal{R}$-invariant iff $x \in A \Rightarrow$ $\mathcal{R}_{x} \subseteq A$ (where $\mathcal{R}_{x}$ is the equivalence class of $x$ ). The collection of $\mathcal{R}$-invariant sets forms a $\sigma$-algebra, which is denoted by $\mathfrak{I}(\mathcal{R})$. A measure $\mu$ is $\mathcal{R}$-ergodic iff every $A \in \mathfrak{I}(\mathcal{R})$ is equal to $\emptyset$ or $X$ up to a $\mu$-null set. We write in this case $\mathfrak{I}(\mathcal{R}) \stackrel{\mu}{=}\{\varnothing, X\}$.

A mixture of measures is a measure $\mu \in \mathfrak{M}(X)$ of form

$$
\mu(B)=\int_{\Omega} \mu_{\omega}(B) \mathrm{d} v(\omega) \quad \forall B \in \mathcal{B}(X)
$$

where $(\Omega, \mathcal{F}, \nu)$ is a $\sigma$-finite measure space, $\mu_{\omega} \in \mathfrak{M}(X) \forall \omega \in \Omega$ and $\omega \mapsto \mu_{\omega}$ is measurable $(\Omega \rightarrow \mathfrak{M}(X))$ in the sense that $\omega \mapsto \mu_{\omega}(A)$ is measurable $\forall A \in \mathcal{B}(X)$.

The mixture is called finite or infinite according to whether $\nu(\Omega)<\infty$ or $v(\Omega)=\infty$, and an average if $\nu(\Omega)=1$. Note that an average of finite measures could also be an infinite mixture of probabilities (see the examples after Proposition 2.2). The measures $\left\{\mu_{\omega}: \omega \in \Omega\right\}$ are called components of (the decomposition of) $\mu$ (note that components are only specified up to $\nu$-measure zero).

If $\mathcal{R}$ is a countable Borel equivalence relation, then any $\mathcal{R}$-invariant $\mu \in \mathfrak{M}(X)$ is an average of $\mathcal{R}$-invariant, ergodic, $\sigma$-finite measures [14].

This reduces the problem of classifying $\mathcal{E}(\Omega)$-invariant measures to that of classifying ergodic $\mathcal{E}(\Omega)$-invariant measures.

Inducing and invariant measures. The following result is a useful property of ergodic measures:

Proposition 1.0. If $A \in \mathcal{B}$ and $\mu \in \mathfrak{M}(A)$ is $\mathcal{R}(A) \equiv \mathcal{R} \cap(A \times A)$-invariant and ergodic, then there is a unique $\bar{\mu} \in \mathfrak{M}(X), \mathcal{R}$-invariant and ergodic, such that $\left.\bar{\mu}\right|_{A} \equiv \mu$.

Proof. Let $\mathcal{R}=\mathcal{R}_{\Gamma}$ where $\Gamma$ is a countable group of automorphisms which generates $\mathcal{R}$ as in [10]. There are $\gamma_{n} \in$ $\Gamma(n \geqslant 1)$ and $A_{n} \in \mathcal{B}(A)$ so that $\bigcup_{\gamma \in \Gamma} \gamma A=\biguplus_{n \geqslant 1} \gamma_{n} A_{n}$. The required measure is $\bar{\mu}(B):=\sum_{n=1}^{\infty} \mu\left(\gamma_{n}^{-1}(B \cap\right.$ $\left.\left.\gamma_{n} A_{n}\right)\right)$.

Conformal measures for tail relations. The conformality property can be significantly simplified when the underlying equivalence relation is a tail relation. Call $\mu \in \mathfrak{M}(X)\left(\mathrm{e}^{F}, T\right)$-conformal if $\mu$ is $T$-nonsingular and $\frac{\mathrm{d} \mu \circ T}{\mathrm{~d} \mu}=\mathrm{e}^{F}$. The following proposition relates this notion to conformality w.r.t. $\mathfrak{T}(T)$ and $\mathfrak{G}(T)$ :

Proposition 1.1. Let $(X, T)$ be a measurable, locally invertible transformation of a measurable space, let $F: X \rightarrow \mathbb{R}$ be measurable and suppose $\widehat{F}$ is as in $(0.1)$.

(1) $\mu \in \mathfrak{M}(X)$ is $\left(\mathrm{e}^{\widehat{F}}, \mathfrak{G}(T)\right)$-conformal iff it is $T$-nonsingular, its support is $\mathfrak{G}(T)$-invariant and $\frac{\mathrm{d} \mu \circ T}{\mathrm{~d} \mu}=\mathrm{e}^{F}$;

(2) If $\mu \in \mathfrak{M}(X)$ is $\mathfrak{G}(T)$-nonsingular, and $\frac{\mathrm{d} \mu \circ T}{\mathrm{~d} \mu}=c \mathrm{e}^{F}$ for some constant $c>0$, then $\mu$ is $\left(\mathrm{e}^{\widehat{F}}, \mathfrak{T}(T)\right)$-conformal.

(3) If $\mu \in \mathfrak{M}(X)$ is $\left(\mathrm{e}^{\widehat{F}}, \mathfrak{T}(T)\right)$-conformal, $\mathfrak{T}(T)$-ergodic, and $\mathfrak{G}(T)$-nonsingular, then $\exists c>0$ constant such that $\frac{\mathrm{d} \mu \circ T}{\mathrm{~d} \mu}=c \mathrm{e}^{F} \bmod \mu$. 
Proof. The first two statements are established by direct calculation. We prove the third. By assumption, for any $\mathfrak{T}(T)$ holonomy $K, \frac{\mathrm{d} \mu \circ K}{\mathrm{~d} \mu}(x)=\mathrm{e}^{\widehat{F}(x, K x)}$. But also, $\frac{\mathrm{d} \mu \circ K}{\mathrm{~d} \mu}(x)=\mathrm{e}^{\left.\widehat{\log T^{\prime}}(x, K x)\right)}$, where $T^{\prime}:=\frac{\mathrm{d} \mu \circ T}{\mathrm{~d} \mu}$. It follows that $\widehat{F} \equiv \widehat{\log T^{\prime}}$ on $\mathfrak{T}(T)$.

We claim that $h:=F-\log T^{\prime}$ is $\mathfrak{T}(T)$-invariant, whence constant $\mu$-a.e. To see this, note first that $\widehat{h}=\widehat{F}-$ $\widehat{\log T^{\prime}} \equiv 0$ on $\mathfrak{T}(T)$. For $(x, y) \in \mathfrak{T}(T)$, we have that $h_{n}(x)=h_{n}(y)$ whenever $T^{n} x=T^{n} y$. If $T^{n} x=T^{n} y$, then also $T^{n+1} x=T^{n+1} y$ and $h(x)=h_{n+1}(x)-h_{n}(T x)=h_{n+1}(y)-h_{n}(T y)=h(y)$. Thus $\frac{\mathrm{d} \mu \circ T}{\mathrm{~d} \mu}=c \mathrm{e}^{F}$ for some $c>0$.

Finally, here is a straightforward generalization of a calculation done in Section 0. Define

$$
\begin{aligned}
& \mathfrak{T}(T, F):=\left\{(x, y) \in X^{2}: \exists n \geqslant 0 \text { s.t. } T^{n} x=T^{n} y, F_{n}(x)=F_{n}(y)\right\} ; \\
& \mathfrak{G}(T, F):=\left\{(x, y) \in X_{0}^{2}: \exists k, \ell \geqslant 0 \text { s.t. } T^{k} x=T^{\ell} y, F_{k}(x)=F_{\ell}(y)\right\} .
\end{aligned}
$$

Proposition 1.2. Let $\mathbb{G}$ be an Abelian topological group and $F: X \rightarrow \mathbb{G}$ measurable. If $\mu \in \mathfrak{M}(X)$ is $\mathfrak{G}(T)$-nonsingular and $\frac{\mathrm{d} \mu \circ T}{\mathrm{~d} \mu}=c \mathrm{e}^{H \circ F} \bmod \mu$ where $H: \mathbb{G} \rightarrow \mathbb{R}$ is a homomorphism and $c>0$, then $\mu$ is $\mathfrak{T}(T, F)$-invariant.

The proof of Proposition 1.2 is immediate from the definitions. As we shall see, much of this paper is boils down to its converses.

The Glimm-Effros theorem. Consider as an example the one-sided two shift, i.e. the map $T$ on $\Omega:=\{0,1\}^{\mathbb{N}}$ defined by $T\left(x_{1}, x_{2}, \ldots\right)=\left(x_{2}, x_{3}, \ldots\right)$. As is well known, there exists a unique $\mathfrak{T}(T)$-invariant probability measure: the $\left(\frac{1}{2}, \frac{1}{2}\right)$-Bernoulli measure. Nevertheless, there are uncountably many non-atomic mutually singular $\mathfrak{T}(T)$-ergodic and invariant $\sigma$-finite measures: Pick $K \subset \mathbb{N}$ with infinite complement and consider the probability measure $\mu_{K}$ on $\Omega_{K}:=\left\{x \in\{0,1\}^{\mathbb{N}}: i \in K \Rightarrow x_{i}=0\right\}$ obtained from the $\left(\frac{1}{2}, \frac{1}{2}\right)$-Bernoulli measure after the identification $\Omega_{K} \simeq$ $\{0,1\}^{\mathbb{N} \backslash K}$. Now extend it to a $\sigma$-finite ergodic invariant measure on $\{0,1\}^{\mathbb{N}}$ using Proposition 1.0.

The same phenomena occurs for a general countable Borel equivalence relation, as soon as it admits a non-atomic ergodic invariant measure, as explained below. Recall that any countable Borel equivalence relation is of the form $\mathcal{R}_{\Gamma}:=\{(x, g(x)): x \in X, g \in \Gamma\}$ for some countable group of Borel automorphisms. We have:

Glimm-Effros theorem. $[9,13]$ Let $\Gamma$ be a countable group of Borel automorphisms of the standard Borel space $X$, then either (i) $X=\biguplus_{\gamma \in \Gamma} \gamma A$ for some $A \in \mathcal{B}$, or (ii) $\exists A \in \mathcal{B}$ so that $A \cong\{0,1\}^{\mathbb{N}}$ and $\mathcal{R}_{\Gamma} \cap A \times A \cong \mathfrak{T}(T)$ where $T$ is the shift on $\{0,1\}^{\mathbb{N}}$.

If $\mathcal{R}$ has at least one non-atomic ergodic non-singular measure, then case (i) cannot occur, and case (ii) must hold. But in this case there are uncountably many mutually singular non-atomic $\sigma$-finite ergodic invariant measures, because of the construction sketched above (see also [26]).

\section{Fibred systems and exchangeability}

Fibred systems. It is convenient to work with measurable fibred systems, because this setting allows to describe countable Markov shifts, $\beta$-expansions, and many other subshifts easily.

A (measurable) fibred system is a triple $(X, T, \alpha)$ where $X$ is a standard measurable space, $T: X \rightarrow X$ is a measurable transformation and $\alpha \subset \mathcal{B}(X)$ is a finite or countable partition such that:

(1) $\bigvee_{i=0}^{\infty} T^{-i} \alpha$ generates $\mathcal{B}$;

(2) for every $A \in \alpha,\left.T\right|_{A}: A \rightarrow T A$ is bi-measurable, invertible.

If $(X, T, \alpha)$ is a fibred system, then for each $k \in \mathbb{N},\left(X, T, \alpha_{k}\right)$ and $\left(X, T^{k}, \alpha_{k}\right)$ are also fibred systems, where

$$
\alpha_{k}:=\bigvee_{j=0}^{k-1} T^{-j} \alpha=\left\{\bigcap_{j=0}^{k-1} T^{-j} a_{j}: a_{0}, \ldots, a_{k-1} \in \alpha\right\}
$$

The elements of $\alpha_{k}$ are called cylinders (of length $k$ ) and are denoted $\bigcap_{j=0}^{k-1} T^{-j} a_{j}=:\left[a_{0}, a_{1}, \ldots, a_{k-1}\right]$. 
If $S$ is countable and $X \subset S^{\mathbb{N}}$ is a subshift, then $(X, T, \alpha)$ is a fibred system where $T$ is the shift and $\alpha:=$ $\left\{a_{s}: s \in S\right\}, a_{s}:=\left\{x \in X: x_{1}=s\right\}$. Here we abuse notation and denote $a_{s}=[s]$.

Let $Y$ be a set. The memory of a function $f: X \rightarrow Y$ is the minimum $k \in \mathbb{N} \cup\{0\}$ so that $f$ is $\alpha_{k+1}$-measurable (i.e. constant on each $a \in \alpha_{k+1}$ ); A function is said to have infinite memory if it is not $\alpha_{k}$-measurable for any $k \geqslant 1$.

Let $(Y, d)$ be a metric space. For $N \geqslant 1$, we say that $\phi: X \rightarrow Y$ is $(\alpha, N)$-Hölder continuous on $A \subset X$ if $\exists \rho \in$ $(0,1), M>0$ such that $x, y \in A \cap \alpha_{k} \Rightarrow d(\phi(x), \phi(y)) \leqslant M \rho^{k}$ for all $k \geqslant N$.

We call the fibred system $(X, T, \alpha)$ full if $T A=X \forall A \in \alpha$ and we call a probability $m \in \mathcal{P}(X)$ a $(X, T, \alpha)$-product measure if

$$
m\left(\bigcap_{j=0}^{n} T^{-j} A_{j}\right)=\prod_{j=0}^{n} m\left(A_{j}\right) \quad \forall n \geqslant 0, A_{0}, \ldots, A_{n} \in \alpha .
$$

Given a fibred system $(X, T, \alpha)$, we define $\pi: X \rightarrow \alpha^{\mathbb{N}}$ by $T^{n-1} x \in \pi(x)_{n} \in \alpha$ and consider the subshift $\Sigma(X, T, \alpha):=\overline{\pi(X)} \subset \alpha^{\mathbb{N}}$. The map $\pi: X \rightarrow \alpha^{\mathbb{N}}$ is injective, and we denote $x=\left(a_{0}, a_{1}, \ldots\right)$ where $\pi(x)=$ $\left(a_{0}, a_{1}, \ldots\right)$. Eventually periodic points of form $p=\left(a_{0}, a_{1}, \ldots, a_{n}, b_{1}, \ldots, b_{k}, b_{1}, \ldots, b_{k}, \ldots\right)$ are denoted $p=$ $\left(a_{0}, a_{1}, \ldots, a_{n}, \overline{b_{1}, \ldots, b_{k}}\right)$. is

The exchangeable relation of a fibred system. The exchangeable equivalence relation of a fibred system $(X, T, \alpha)$

$$
\mathcal{E}(X, T, \alpha):=(\pi \times \pi)^{-1} \mathcal{E}(\Sigma(X, T, \alpha)) .
$$

As before $\mathcal{E}(X, T, \alpha)=\mathfrak{T}\left(T, F^{\natural}\right)$, where

$$
F^{\natural}=F^{\natural, a_{0}}: X \rightarrow \mathbb{Z}_{0}^{\alpha \backslash\left\{a_{0}\right\}}, \quad F^{\natural}(x)_{a}:=1_{a}(x) \quad\left(a \in \alpha \backslash a_{0}\right),
$$

$a_{0} \in \alpha$ is fixed, and $\mathbb{Z}_{0}^{\alpha \backslash\left\{a_{0}\right\}}:=\left\{x \in \mathbb{Z}^{\alpha \backslash\left\{a_{0}\right\}}: \#\left\{s \in \alpha: x_{s} \neq 0\right\}<\infty\right\}$ equipped with the discrete topology and vector addition.

We note for future reference that $\left\{F^{\natural}(x)-F^{\natural}(y): x, y \in X\right\}$ generates $\mathbb{Z}_{0}^{\alpha \backslash\left\{a_{0}\right\}}$ (this would not have been true had we worked with $\mathbb{Z}_{0}^{S}$ ). We also note that $F^{\natural}$ is $\alpha$-measurable.

Conformal measures for fibred systems. Recall that our plan is to relate exchangeable measures to $\left(\mathfrak{T}(T), \mathrm{e}^{H \circ F^{\natural}}\right)$ conformal measures for some continuous homomorphism $H: \mathbb{Z}_{0}^{S \backslash\left\{a_{0}\right\}} \rightarrow \mathbb{R}$. The following proposition relates conformality with the property of having $\alpha$-measurable derivative (compare with Proposition 1.1 above):

Proposition 2.0. Let $\mu \in \mathfrak{M}_{\alpha}(X)$ be $\mathfrak{G}(T)$-non-singular, then

(1) $\frac{\mathrm{d} \mu \circ T}{\mathrm{~d} \mu}$ is $\alpha$-measurable $\Leftrightarrow \frac{\mathrm{d} \mu \circ T}{\mathrm{~d} \mu}=c \mathrm{e}^{H \circ F^{\natural}}$ for some $c>0$ and some homomorphism $H: \mathbb{Z}_{0}^{\alpha \backslash\left\{a_{0}\right\}} \rightarrow \mathbb{R}$; and

(2) in this case, $\mu$ is $\mathcal{E}(X, T, \alpha)$-invariant.

Proof. The $(\Leftarrow)$ implication in $(1)$ is clear. We prove $(\Rightarrow)$ : Let $c$ be the value of $\frac{\mathrm{d} \mu \circ T}{\mathrm{~d} \mu}$ on $a_{0}$, and define a homomorphism $H: \mathbb{Z}_{0}^{\alpha \backslash\left\{a_{0}\right\}} \rightarrow \mathbb{R}$ by $H\left(e_{a}\right):=\left.\log \frac{\mathrm{d} \mu \circ T}{\mathrm{~d} \mu}\right|_{a}-\log c$ (this is a constant) where for every $a \in \alpha,\left(e_{a}\right)_{b}=\delta_{a, b}$ $(b \in \alpha)$. We have $\frac{\mathrm{d} \mu \circ T}{\mathrm{~d} \mu}=c \mathrm{e}^{H \circ F^{\natural}}$. Part (2) follows from Proposition 1.2.

Recurrence and the de Finetti-Hewitt-Savage theorem. The de Finetti-Hewitt-Savage theorem states:

Theorem 2.1. Let $(X, T, \alpha)$ be a full fibred system.

1. $\mathcal{E}(X, T, \alpha)$ is ergodic with respect to any $(X, T, \alpha)$-product measure.

2. If $\mu \in \mathcal{P}(X)$ is $\mathcal{E}(X, T, \alpha)$-invariant, then $\mu$ is an average of $(X, T, \alpha)$-product probability measures.

3. If $\mu \in \mathcal{P}(X)$ is $\mathcal{E}(X, T, \alpha)$-invariant, then $\mathfrak{I}(\mathcal{E}(X, T, \alpha)) \stackrel{\mu}{=} \mathfrak{I}(\mathfrak{T}(T))$.

Proof. See [16] (also [20,8]). 
We will need to consider extensions of part (2) of the theorem for $\sigma$-finite measures.

For this purpose we need the following definitions. Suppose $(X, T, \alpha)$ is a fibred system, and define for every $a \in \alpha$.

$$
N_{a}:=\sum_{n=0}^{\infty} 1_{a} \circ T^{n} .
$$

A measure $\mu \in \mathfrak{M}(X)$ is called recurrent (w.r.t. to $(X, T, \alpha))$ if $N_{a} \in\{0, \infty\} \mu$-a.e. for every $a \in \alpha$.

Proposition 2.2. Let $(X, T, \alpha)$ be a full fibred system, and suppose $\mu \in \mathfrak{M}(X)$ is $\mathcal{E}(X, T, \alpha)$-invariant.

(1) If $\mu$ is topologically $\sigma$-finite and recurrent, then $\mu$ is a mixture of $(X, T, \alpha)$-product probability measures.

(2) If $\mu$ is locally finite, then $\mu$ is recurrent, whence by (1) a mixture of $(X, T, \alpha)$-product probability measures.

Proof. Suppose $\mu$ is topologically $\sigma$-finite and recurrent $\mathcal{E}$-invariant measure, where $\mathcal{E}:=\mathcal{E}(X, T, \alpha)$. For every $x \in X$, define $S_{x}:=\left\{x_{n}: n \geqslant 1\right\}$. By recurrence, $\forall s \in S_{x}, N_{s}(x)=\infty$ for $\mu$-a.e. $x \in X$.

First, we consider possible atoms of $\mu$. Suppose $x=\left(s_{1}, s_{2}, \ldots\right) \in X$ is an atom. We claim that $s_{n}=s_{1} \forall n \geqslant 1$. Otherwise, fix (using topological $\sigma$-finiteness and $\mu\{x\} \neq 0$ ) some $N$ such that $\left[s_{1}, \ldots, s_{N}\right]$ has finite measure. Since $\mu$ is recurrent $\left|S_{T^{N}}\right|=\left|S_{x}\right|>1$, and consequently $\left(s_{k}\right)_{k \geqslant N}$ has an infinite number of finite permutations $\{y(k)\}_{k \geqslant 1}$. Using the assumption that $(X, T, \alpha)$ is full, we see that $z(k):=\left(s_{1}, \ldots, s_{N}, y(k)\right) \in X \cap\left[s_{1}, \ldots, s_{N}\right]$. By exchangeability, $\mu\{z(k)\}=\mu\{x\} \neq 0$. But this implies that $\mu\left[s_{1}, \ldots, s_{N}\right]=\infty$, a contradiction.

The conclusion is that any atom of $\mu$ must be of the form $x=(s, s, \ldots)$. If $x=(s, s, \ldots)$ then $\delta_{x}$ is an atomic $(X, T, \alpha)$-product measure. Thus $v$ is a mixture of atomic $(X, T, \alpha)$-product measures and a non-atomic, topologically $\sigma$-finite, recurrent and $\mathcal{E}$-invariant measure. This allows us to assume (as we do henceforth) that $\mu$ is non-atomic.

By the ergodic decomposition (see [14]), $\mu$ is an average of $\mathcal{E}$-invariant, ergodic components, each of which is nonatomic, recurrent and topologically $\sigma$-finite. We claim each component is a multiple of a $(X, T, \alpha)$-product measure.

Accordingly, assume that $\mu \in \mathfrak{M}(X)$ is topologically $\sigma$-finite, recurrent and $\mathcal{E}$-invariant and ergodic. For each $s \in S,\left\{x \in X: s \in S_{x}\right\} \in \mathfrak{I}(\mathcal{E})$, whence $\exists S^{\prime} \subseteq S$ such that $S_{x}=S^{\prime}$ a.e. We assume (without loss of generality) that $S^{\prime}=S$.

We claim that $\mu(a)>0, \forall a \in \alpha_{k}, k \geqslant 1$. To see this, let $a=\left[a_{1}, \ldots, a_{k}\right] \in \alpha_{k}$, set $S_{a}:=\left\{a_{1}, \ldots, a_{k}\right\}$, and let $n_{s}(a)$ denote the number of times $s$ appears in $a$. Consider the collection $\mathcal{K}_{+}^{*}$ of all families of disjoint subsets of $\mathbb{N}$ $\left\{K_{s}: s \in S_{a}\right\}$ such that $\left|K_{s}\right|=n_{s}(a)$ for all $s$. For every $\mathcal{K}_{+} \in \mathcal{K}_{+}^{*}$, set

$$
A\left(\mathcal{K}_{+}\right):=\left\{x=\left(x_{1}, x_{2}, \ldots\right) \in X: x_{n}=s \forall n \in K_{s}, s \in S_{a}\right\} .
$$

By recurrence, $X \stackrel{\bmod \mu}{=}\left[N_{s} \geqslant n_{s}(a) \forall s \in S_{a}\right]=\bigcup_{\mathcal{K}_{+} \in \mathcal{K}_{+}^{*}} A\left(\mathcal{K}_{+}\right)$. Since $\mathcal{K}_{+}^{*}$ is countable, we can choose $\mathcal{K}_{+} \in \mathcal{K}_{+}^{*}$ s.t. $\mu\left[A\left(\mathcal{K}_{+}\right)\right] \neq 0$. Evidently $A\left(\mathcal{K}_{+}\right) \stackrel{\mathcal{E}}{\longrightarrow} a$. Since $\mu$ is exchangeable, $\mu(a)>0$.

Now let $\beta$ be the collection of all cylinders of positive finite measure. For each $a \in \beta \cap \alpha_{n}$, define $v_{a} \in \mathcal{P}(X)$ by $v_{a}(B):=\frac{\mu\left(a \cap T^{-n} B\right)}{\mu(a)}$, then $v_{a}$ is $\mathcal{E}$-invariant, ergodic and by Theorem 2.1 (part 2), a $(X, T, \alpha)$-product measure.

We claim that $v_{a}$ does not depend on $a \in \beta$. To see this note that for $a \in \beta, b \in \alpha_{n}$ (some $n \geqslant 1$ ) we have $a b \in \beta$ and for $c \in \alpha_{k}$,

$$
v_{a b}(c)=\frac{\mu(a b c)}{\mu(a b)}=\frac{\mu(a) v_{a}(b) v_{a}(c)}{\mu(a) v_{a}(b)}=v_{a}(c) .
$$

Thus for $a, b \in \beta, c \in \alpha_{k}$, we have $a b \stackrel{\mathcal{E}}{\longrightarrow} b a, a b c \stackrel{\mathcal{E}}{\longrightarrow} b a c$, whence

$$
v_{a}(c)=v_{a b}(c)=\frac{\mu(a b c)}{\mu(a b)}=\frac{\mu(b a c)}{\mu(b a)}=v_{b a}(c)=v_{b}(c) .
$$

Writing $v_{a}=v(a \in \beta)$, we see that for $a, b \in \beta$ :

$$
\mu(a) v(b)=\mu(a b)=\mu(b a)=\mu(b) v(a) .
$$

Fixing $\beta_{0} \subset \beta$ so that $X=\biguplus_{b \in \beta_{0}} b \bmod \mu$ and fixing $a \in \beta, v(a)>0$, we have

$$
\mu(X)=\sum_{b \in \beta_{0}} \mu(b)=\sum_{b \in \beta_{0}} \frac{\mu(a) v(b)}{v(a)} \leqslant \frac{\mu(a)}{v(a)}<\infty
$$


and part 1 follows from part 2 of Theorem 2.1.

In order to prove part 2, assume by way of contradiction that $\mu$ is locally finite, but not recurrent. Fix $a \in S$ and $N \in \mathbb{N}$ so that $\mu\left(\left[N_{a}=N\right]\right)>0$. By local finiteness, $\exists$ a cylinder $b:=\left[b_{1}, \ldots, b_{K}\right]$ with $0<\mu\left(b \cap\left[N_{a}=N\right]\right)<\infty$ and $\#\left\{1 \leqslant i \leqslant K: b_{i}=a\right\}=N . \exists \sigma$ a permutation so that

$$
B:=\left[b_{\sigma(1)}, \ldots, b_{\sigma(K)}\right]=[\underbrace{a, \ldots, a}_{N \text {-times }}, B_{N+1}, \ldots, B_{K}] .
$$

By exchangeability, $0<\mu\left(B \cap\left[N_{a}=N\right]\right)=\mu\left(b \cap\left[N_{a}=N\right]\right)<\infty$. By local finiteness, we may assume $0<$ $\mu\left(\left[B_{N+1}, \ldots, B_{K}\right]\right)<\infty$.

For $n \in \mathbb{N}$, define $\kappa_{n}: B \cap\left[N_{a}=N\right] \rightarrow\left[B_{N+1}, \ldots, B_{K}\right] \cap\left[N_{a}=N\right]$ by

$$
\kappa_{n}(\underbrace{a, \ldots, a}_{N \text {-times }}, B_{N+1}, \ldots, B_{K}, x_{1}, x_{2}, \ldots):=(B_{N+1}, \ldots, B_{K}, x_{1}, \ldots, x_{n}, \underbrace{a, \ldots, a}_{N \text {-times }}, x_{n+1}, \ldots) .
$$

But $\mu\left(\kappa_{n}\left(B \cap\left[N_{a}=N\right]\right)\right)=\mu\left(B \cap\left[N_{a}=N\right]\right)>0 \forall n \in \mathbb{N}$, in contradiction to $0<\mu\left(\left[B_{N+1}, \ldots, B_{K}\right]\right)<\infty$ as $\left[B_{N+1}, \ldots, B_{K}\right] \supseteq \biguplus_{n \geqslant 1} \kappa_{n}\left(B \cap\left[N_{a}=N\right]\right)$.

Examples. Consider $X=\{0,1\}^{\mathbb{N}}$ with $T$ the shift and $\alpha=\{0,1\}$.

(1) The mixture above may not be finite: Set $\mu_{p}:=\prod\left((1-p) \delta_{0}+p \delta_{1}\right)$, then the measure $\mu:=\sum_{n=1}^{\infty} \frac{1}{n+1} \mu_{1 / n}$ is a $\sigma$-finite, infinite mixture of $(X, T, \alpha)$-product measures. If $v_{n}:=n \mu_{1 / n}$, then $\mu:=\sum_{n=1}^{\infty} \frac{1}{n(n+1)} v_{n}$ is an average and $\mu([1])=v_{n}([1])=1 \forall n \geqslant 1$.

(2) The recurrence condition cannot be removed: Let $e_{n} \in X,\left(e_{n}\right)_{i}=0 \forall i \neq n,\left(e_{n}\right)_{n}=1$, then $\mu:=\sum_{n=1}^{\infty} \delta_{e_{n}} \in$ $\mathfrak{M}(X)$ is $\mathcal{E}(X, T, \alpha)$-invariant, ergodic but not a mixture of $(X, T, \alpha)$-product measures. This example is topologically $\sigma$-finite, but not locally finite, or recurrent.

(3) Comparison with the Glimm-Effros theorem: Let $X_{\infty}:=\left\{x \in X: N_{0}(x)=N_{1}(x)=\infty\right\} \in \mathcal{B}(X)$. By Theorem 2.1, there are non-atomic $\mathcal{E}(X, T, \alpha) \cap\left(X_{\infty} \times X_{\infty}\right)$-invariant, ergodic probabilities. Thus, by the Glimm-Effros theorem (above), there are uncountably many mutually singular, non-atomic, infinite, $\sigma$-finite, recurrent, $\mathcal{E}(X, T, \alpha)$ invariant, ergodic measures on $X$. Proposition 2.2 says that these measures cannot be topologically $\sigma$-finite.

\section{Part 2. Exchangeability for topological Markov shifts}

\section{Topological Markov shifts and existence of conformal measures}

Topological Markov shifts. A topological Markov shift (TMS) is a fibred system $(X, T, \alpha)$ which satisfies the Markov property - for every $A \in \alpha, T(A)$ is $\alpha$-measurable - and whose topology is generated by the set of cylinders.

The elements of $\alpha$ are called the states of the shift, and the matrix $\left(A_{a b}\right)_{\alpha \times \alpha}$ with $A_{a b}=1$ if $[a, b] \neq \emptyset$ and $A_{a b}=0$ otherwise is called the transition matrix of the shift. A TMS with set of states $\alpha$ and transition matrix $\left(t_{a b}\right)_{\alpha \times \alpha}$ can be canonically identified with the set $\left\{x=\left(x_{1}, x_{2}, \ldots\right): \forall i t_{x_{i} x_{i+1}}=1\right\}$ together with the action of the left shift and the relative product topology in $\alpha^{\mathbb{N}}$.

A topological Markov shift is topologically transitive is for any $a, b \in \alpha$ there exists some $n$ such that $a \cap T^{-n} b \neq \emptyset$, and topologically mixing (or simply mixing) if for all $a, b \in \alpha, a \cap T^{-n} b \neq \emptyset$ for all $n$ sufficiently large. (These definitions agree with the standard definitions of these notions for topological dynamical systems.)

A topological Markov shift is said to be almost onto [4], if $\forall b, c \in \alpha, \exists n \geqslant 1, b=a_{0}, a_{1}, \ldots, a_{n}=c \in \alpha$ such that $T a_{k} \cap T a_{k+1} \neq \emptyset(0 \leqslant k \leqslant n-1)$. This property comes up in the study of ergodicity done in Section 4 .

The following example (Example 5.2 in [23]) shows that mixing $\nRightarrow$ almost onto. Let $X \subset\{0,1,2\}^{\mathbb{N}}$ be the TMS with transition matrix

$$
A:=\left(\begin{array}{lll}
1 & 1 & 0 \\
0 & 0 & 1 \\
1 & 1 & 0
\end{array}\right) .
$$

Evidently, $(X, T, \alpha)$ is mixing, but not almost onto. 
Existence of conformal measures. As we shall see below (Theorems 5.0, 5.1, and 5.2) the conformal measures which appear in the study of exchangeability for TMS satisfy the conditions of Proposition 2.0, and therefore have $\alpha$-measurable derivatives. It is therefore enough to clarify what $\alpha$-measurable functions can appear as derivatives. This is done in the next proposition:

Proposition 3.0. Let $(X, T, \alpha)$ be a mixing TMS with countable state space $S$ and let $\pi: S \rightarrow \mathbb{R}^{+}$. There is a $\mu \in$ $\mathfrak{M}_{\alpha}(X)$ with $\frac{\mathrm{d} \mu \circ T}{\mathrm{~d} \mu} \alpha$-measurable and $\mu([s])=\pi_{s}(s \in S) \Leftrightarrow \Pi(s):=\sum_{t \in S} A_{s, t} \pi_{t}<\infty \forall s \in S$. In this case, the measure $\mu$ is a Markov measure, and is unique.

Proof. It is easy to see that $\frac{\mathrm{d} \mu \circ T}{\mathrm{~d} \mu}$ is $\alpha$-measurable iff $\mu$ is a Markov measure of the form $\mu\left(\left[s_{1}, s_{2}, \ldots, s_{n}\right]\right)=$ $\pi_{s_{1}} p_{s_{1}, s_{2}} \ldots p_{s_{n-1}, s_{n}}$, where

$$
p_{s, t}=\frac{A_{s, t} \pi_{t} h(s)}{\pi_{s}} \quad \text { and } \quad \sum_{t \in S} A_{s, t} \pi_{t}=\frac{\pi_{s}}{h(s)} \quad \forall s \in S \text { and some } h: S \rightarrow \mathbb{R}_{+} .
$$

The first condition implies that $\frac{\mathrm{d} \mu \circ T}{\mathrm{~d} \mu}(x)=\frac{\pi_{x_{2}}}{\pi_{x_{1}} p_{x_{1}, x_{2}}}=\frac{1}{h\left(x_{1}\right)}$; The second guarantees that $\left(p_{s, t}\right)$ is a stochastic matrix.

We prove the equivalence proclaimed above.

$(\Rightarrow)$ Suppose that $\mu\left(\left[s_{1}, s_{2}, \ldots, s_{n}\right]\right)=\pi_{s_{1}} p_{s_{1}, s_{2}} \cdots p_{s_{n-1}, s_{n}}$ where $p_{s, t}=\frac{A_{s, t} \pi_{t} h(s)}{\pi_{s}}$ for some $h: S \rightarrow \mathbb{R}_{+}$, then $\Pi(s):=\sum_{t \in S} A_{s, t} \pi_{t}=\sum_{t \in S} \frac{\pi_{s} p_{s, t}}{h(s)}=\frac{\pi_{s}}{h(s)}<\infty$.

$(\Leftarrow)$ Set $\mu\left(\left[s_{1}, s_{2}, \ldots, s_{n}\right]\right):=\pi_{s_{1}} p_{s_{1}, s_{2}} \cdots p_{s_{n-1}, s_{n}}$ where $p_{s, t}=\frac{A_{s, t} \pi_{t}}{h(s) \pi_{s}}$ and $h(s):=\frac{\Pi(s)}{\pi_{s}}$. This is a stochastic matrix: $\sum_{t \in S} p_{s, t}=\sum_{t \in S} \frac{A_{s, t} \pi_{t}}{h(s) \pi_{s}}=\frac{\Pi(s)}{h(s) \pi_{s}}=1$.

Remarks. It is not clear when the measures in Proposition 3.0 are recurrent. However:

(1) We give examples of recurrent measures with $\alpha$-measurable derivatives on simple aperiodic random walks below.

(2) Let $(X, T, \alpha)$ be a mixing TMS with state space $S$. Using the methods of [29], one can characterize those $h: S \rightarrow \mathbb{R}^{+}$for which there is a recurrent $v \in \mathfrak{M}_{\alpha}(X)$ with $\frac{\mathrm{d} v \circ T}{\mathrm{~d} v}(x)=\frac{c}{h\left(x_{0}\right)}$ for some $c>0$. The condition is that $\exists s \in S$ for which the power series

$$
F(x):=\sum_{n=1}^{\infty} z^{n} \sum_{x \in[s], T^{n} x=x} h\left(x_{0}\right) h\left(x_{1}\right) \cdots h\left(x_{n-1}\right)
$$

has a positive radius of convergence $R_{S}$ and $F\left(R_{S}\right)=\infty$.

(3) As shown in [25], the previous condition always holds if $\sum_{s \in S} h(s)<\infty$ and $(X, T, \alpha)$ has the big images and preimages (BIP) property: $\exists b_{1}, \ldots, b_{N} \in S$ such that $\forall a \exists i, j$ such that $A_{b_{j} a} A_{a b_{i}}=1$.

\section{4. $\mathcal{E}(X, T, \alpha)$-Ergodicity of measures with $\alpha$-measurable derivatives}

Topological transitivity and aperiodicity. An equivalence relation on a topological space is called topologically transitive, if at least one of its equivalence class is dense. This is a necessary condition for ergodicity w.r.t. a globally supported measure. For some classes of measures, it is also a sufficient condition.

We study topological transitivity for $\mathfrak{T}\left(T_{\phi}\right) \equiv \mathfrak{T}(T)_{\widehat{\phi}}$ in the case of a TMS $(X, T, \alpha)$ and an $\alpha$-measurable $\phi: X \rightarrow \mathbb{G}$, where $\mathbb{G}$ denotes a locally compact, Abelian, Polish (LCAP) topological group.

Suppose that $\phi: X \rightarrow \mathbb{G}$ is continuous. For $s \in S n \geqslant 1$, let $\Pi_{n, s}:=\left\{x \in[s]: T^{n} x=x\right\}$, let $\Pi_{n}:=\{x \in X:$ $\left.T^{n} x=x\right\}=\bigcup_{s \in S} \Pi_{n, s}$ and let

$$
\begin{aligned}
& \mathbb{F}_{\phi, s}:=\left\langle\left\{\phi_{n}(x)-\phi_{n}(y): n \geqslant 1, x, y \in \Pi_{n, s}\right\}\right\rangle, \\
& \mathbb{F}_{\phi}:=\left\langle\left\{\phi_{n}(x)-\phi_{n}(y): n \geqslant 1, x, y \in \Pi_{n}\right\}\right\rangle,
\end{aligned}
$$

where $\langle A\rangle$ denotes the subgroup generated by $A$.

Note that if $\phi$ is $\alpha$-measurable, then $\left\{\phi_{n}(x)-\phi_{n}(y): n \geqslant 1, x, y \in \Pi_{n, s}\right\}$ is a group for every $s$. If in addition $(X, T, \alpha)$ is mixing, then $\left\{\phi_{n}(x)-\phi_{n}(y): n \geqslant 1, x, y \in \Pi_{n}\right\}$ is also a group. 
Proposition 4.0. If $(X, T, \alpha)$ is mixing and $\phi: X \rightarrow \mathbb{G}$ is $\alpha$-measurable, then $\mathbb{F}_{\phi, s}=\mathbb{F}_{\phi} \forall s \in S$.

Proof. We first show that $\mathbb{F}_{\phi, s}=\mathbb{F}_{\phi, t} \forall s, t \in S$. To see this fix (using mixing) $k \geqslant 2$, sat, tbs $\in \alpha_{k}$. If $g \in \mathbb{F}_{\phi, s}$, then $\exists n \geqslant 1, \overline{s x}, \overline{s y} \in \Pi_{n, s}$ with $g=\phi_{n}(\overline{s x})-\phi_{n}(\overline{s y})$. It follows that $\overline{t b s x s a}, \overline{t b s y s a} \in \Pi_{t, n+2 k-2}$ and

$$
\phi_{n+2 k-2}(\overline{t b s x s a})-\phi_{n+2 k-2}(\overline{t b s y s a})=\phi_{n}(\overline{s x})-\phi_{n}(\overline{t y})=g \in \mathbb{F}_{\phi, t} .
$$

Now we show that $\mathbb{F}_{\phi, u}=\mathbb{F}_{\phi} \forall u \in S$. Let $g \in \mathbb{F}_{\phi}$, then $\exists n \geqslant 1, s, t \in S, \overline{s x} \in \Pi_{n, s}, \overline{t y} \in \Pi_{n, t}$ with $g=\phi_{n}(\overline{s x})-$ $\phi_{n}(\overline{t y})$. By the previous paragraph, it suffices to show that $g \in \mathbb{F}_{\phi, s}$. To this end, using mixing, fix $k \geqslant 1$, sat, tbs $\in \alpha_{k}$, then $\overline{\text { sxsatb }}, \overline{\text { satytb }} \in \Pi_{s, n+2 k-2}$ and $\phi_{n+2 k-2}(\overline{\text { sxsatb }})-\phi_{n+2 k-2}(\overline{\text { satytb }})=\phi_{n}(\overline{s x})-\phi_{n}(\overline{s y})=g \in \mathbb{F}_{\phi, s}$.

We call the continuous $\phi: X \rightarrow \mathbb{G}$ (topologically) aperiodic if $\overline{\mathbb{F}}_{\phi}=\mathbb{G}$. It follows from Lemma 4.3 (below) that this is equivalent to the absence of non-trivial solutions to the functional equation $\gamma \circ \phi=\lambda \frac{g \circ T}{g}$ where $\gamma \in \widehat{\mathbb{G}}, \lambda \in \mathbb{S}^{1}$ and $g: X \rightarrow \mathbb{S}^{1}$ continuous.

Proposition 4.1 (tail transitivity). If $(X, T, \alpha)$ is mixing and $\phi: X \rightarrow \mathbb{G}$ is $\alpha$-measurable and aperiodic, then $\mathfrak{T}\left(T_{\phi}\right)$ is topologically transitive on $X \times \mathbb{G}$.

Proof. Fix $g \in \mathbb{F}_{\phi}, k \geqslant 1, a, b \in \alpha_{k}$. We will show that $\exists a^{\prime} \subseteq a, b^{\prime} \subseteq b$ so that $a^{\prime} \times\{0\} \stackrel{\mathfrak{T}\left(T_{\phi}\right)}{\rightarrow} b^{\prime} \times\{g\}$.

Indeed, by the mixing of $(X, T, \alpha), \exists \ell \geqslant 1, c, d \in \alpha_{\ell}, s \in S$ so that acs, $b d s \in \alpha_{k+\ell+1}$. By Proposition $4.0, \mathbb{F}_{\phi, s} \ni g$ and $\exists p \geqslant 1, x, y \in \Pi_{p, s}$ so that $\phi_{p}(y)-\phi_{p}(x)=g+\phi_{k+\ell}(a c s)-\phi_{k+\ell}(b d s)$.

It follows that $\left[a c x_{1}^{p} s\right] \times\{0\} \stackrel{\mathfrak{T}\left(T_{\phi}\right)}{\rightarrow}\left[b d y_{1}^{p} s\right] \times\{g\}$. Transitivity now follows from aperiodicity: $\overline{\mathbb{F}}_{\phi}=\mathbb{G}$.

By [3], $(X, T, \alpha)$ is almost onto iff $F^{\natural}: X \rightarrow \mathbb{Z}_{0}^{\alpha \backslash\left\{a_{0}\right\}}$ (with $a_{0} \in \alpha$ fixed, as defined above) is aperiodic. It is also shown there that in this case an $\alpha$-measurable $\phi: X \rightarrow \mathbb{G}$ is aperiodic whenever $\langle\{\phi(x)-\phi(y): x, y \in X\}\rangle$ is dense in $\mathbb{G}$.

To illustrate this, we give an aperiodicity proof that the example given in Section 3 is not almost onto: Define $F^{\natural}: X \rightarrow \mathbb{Z}^{\{1,2\}}$ by $F^{\natural}(x)=e_{x_{1}}$ if $x_{1}=1,2$ and $F^{\natural}(x):=0$ if $x_{1}=0$. We see that $F_{1}^{\natural}=F_{2}^{\natural} \circ T$, whence

$$
F^{\natural}=\left(F_{2}^{\natural}, F_{2}^{\natural}\right)+\left(F_{2}^{\natural} \circ T, 0\right)-\left(F_{2}^{\natural}, 0\right) .
$$

Therefore $F^{\natural}: X \rightarrow \mathbb{Z}_{0}^{\{1,2\}}$ is not aperiodic, and so $X$ cannot be almost onto.

An $\alpha$-measurable $\phi$ which is not aperiodic, can be modified by a coboundary to be aperiodic as a function into a smaller group. We explain how to do this.

Livsic cohomology Theorem 4.2. [18] Suppose that $(X, T, \alpha)$ is a topologically transitive TMS. Let the topology on $\mathbb{G}$ be generated by the norm $\|\cdot\|_{\mathbb{G}}$ and suppose that $N \geqslant 1, \phi: X \rightarrow \mathbb{G}$ is $(\alpha, N)$-Hölder continuous. If $\phi_{n}(x)=0$ $\forall x \in X, T^{n} x=x$, then $\exists g: X \rightarrow \mathbb{G}(\alpha, N)$-Hölder continuous such that $\phi=g-g \circ T$.

Proof. Fix $z \in X$ with $\overline{\left\{T^{n} z: n \in \mathbb{Z}\right\}}=X$, fix $G(z) \in \mathbb{G}$ and define $G:\left\{T^{n} z: n \in \mathbb{Z}\right\} \rightarrow \mathbb{G}$ by $G\left(T^{n} z\right):=G(z)+$ $\phi_{n}(z)$. We'll extend the domain of definition of $G$ to all $X$ and show that the extension is $(\alpha, N)$-Hölder continuous.

Suppose that $\rho \in(0,1)$ and $\|\phi(x)-\phi(y)\| \leqslant M \rho^{n} \forall n \geqslant N, x, y \in X, x_{1}^{n}=y_{1}^{n}$. It suffices to show that

$$
\|G(u)-G(v)\| \leqslant \frac{M \rho^{n}}{1-\rho} \quad \forall n \geqslant N, u, v \in\left\{T^{n} z: n \in \mathbb{Z}\right\}, u_{1}^{n}=v_{1}^{n} .
$$

Accordingly, suppose that $n \geqslant N, \ell>k,\left(T^{k} z\right)_{1}^{n}=\left(T^{\ell} z\right)_{1}^{n}$ and let $y:=\overline{z_{k+1}^{\ell}}$, then $y \in X, T^{\ell-k} y=y$ and $y_{1}^{\ell-k+n}=$ $z_{k+1}^{\ell+n}$. Thus

$$
\begin{aligned}
\left\|G\left(T^{k} z\right)-G\left(T^{\ell} z\right)\right\| & =\left\|\phi_{\ell-k}\left(T^{k} z\right)\right\| \\
& \left.=\left\|\phi_{\ell-k}\left(T^{k} z\right)-\phi_{\ell-k}(y)\right\| \quad \text { (because } \phi_{\ell-k}(y)=0\right)
\end{aligned}
$$




$$
\begin{aligned}
& \leqslant \sum_{i=0}^{\ell-k-1}\left\|\phi\left(T^{k+i} z\right)-\phi\left(T^{i} y\right)\right\| \\
& =M \sum_{i=0}^{\ell-k-1} \rho^{n+\ell-k-i} \quad \text { (because } z_{k+i+1}^{\ell+n}=y_{i+1}^{\ell-k+n} \text { ) } \\
& \leqslant \frac{M \rho^{n}}{1-\rho} .
\end{aligned}
$$

Cohomology Lemma 4.3. (cf. [22]) If $(X, T, \alpha)$ is mixing and $\phi: X \rightarrow \mathbb{G}$ is $\alpha$-measurable, then $\phi=a+g-g \circ T+\bar{\phi}$ where $a \in \mathbb{G}, \bar{\phi}: X \rightarrow \overline{\mathbb{F}}_{\phi}$ and $g: X \rightarrow \mathbb{G}$ are both $\alpha$-measurable such that $\bar{\phi}: X \rightarrow \mathbb{F}_{\phi}$ is aperiodic.

Proof. By assumption, $\forall N \geqslant 1$ such that $\Pi_{N} \neq \emptyset, \exists a_{N} \in \mathbb{G}$ so that $\phi_{N}(x)=a_{N} \bmod \mathbb{F}_{\phi} \forall x \in \Pi_{N}$. Evidently $k a_{N}=$ $N a_{k} \bmod \mathbb{F}_{\phi}$ whence $\left(\phi_{N}-a_{N}\right)_{k}=0 \bmod \mathbb{F}_{\phi}$ whenever $\Pi_{k}, \Pi_{N} \neq \emptyset$.

By Livsic's theorem, $\forall N \geqslant 1$ such that $\Pi_{N} \neq \emptyset, \exists g^{(N)}: X \rightarrow \mathbb{G} \alpha$-measurable such that

$$
\phi_{N}=a_{N}+g^{(N)}-g^{(N)} \circ T \bmod \mathbb{F}_{\phi} .
$$

Since $(X, T, \alpha)$ is mixing, $\exists p, q \in \mathbb{N}$ relatively prime, and $u, v \in X, T^{p} u=u, T^{q} v=v$. Suppose that $k, \ell \in \mathbb{N}$ satisfy $k p-\ell q=1$, then $\bmod \mathbb{F}_{\phi}$,

$$
\begin{aligned}
\phi & =\phi_{k p}-\phi_{\ell q} \circ T \\
& =k a_{p}-\ell a_{q}+g^{(p)}-g^{(p)} \circ T^{k}-g^{(q)} \circ T+g^{(q)} \circ T^{\ell+1} \\
& =k a_{p}-\ell a_{q}+g_{k}^{(p)}-g_{k}^{(p)} \circ T-g_{\ell}^{(q)} \circ T+g_{\ell}^{(q)} \circ T^{2} \\
& =a+g-g \circ T
\end{aligned}
$$

where $a:=k a_{p}-\ell a_{q}$ and $g:=g_{k}^{(p)}-g_{\ell}^{(q)} \circ T$.

Now let $\bar{\phi}:=\phi-(a+g-g \circ T)$, then $\bar{\phi}: X \rightarrow \mathbb{F}_{\phi}$ is $\alpha$-measurable and aperiodic since $\left\langle\bar{\phi}_{n}(x)-\bar{\phi}_{n}(y): n \geqslant 1\right.$, $\left.x, y \in \Pi_{n}\right\rangle=\mathbb{F}_{\phi}$.

Ergodicity. Let $(X, T, \alpha)$ be a TMS and $\mu \in \mathfrak{M}_{\alpha}(X)$ be a $\mathfrak{G}(T)$-nonsingular measure. Consider the following properties:

(1) The Gibbs property: $\frac{\mathrm{d} \mu \circ T}{\mathrm{~d} \mu}$ is $(\alpha, 1)$-Hölder continuous;

(2) The Markovian Gibbs property: $\frac{\mathrm{d} \mu \circ T}{\mathrm{~d} \mu}$ is $(\alpha, 2)$-Hölder continuous.

Some examples: If $(X, T, \alpha)$ is full, then any global $(X, T, \alpha)$-product has the Gibbs property; and if $(X, T, \alpha)$ is a TMS , then any global Markov measure on $X$ has the Markovian Gibbs property.

Lemma 4.4. Suppose that $(X, T, \alpha)$ is full and that $m \in \mathcal{P}(X)$ is a globally supported measure with the Gibbs property so that $(X, \mathcal{B}, m, T)($ where $\mathcal{B}:=\mathcal{B}(X))$ is conservative and exact, then $m$ is $\mathcal{E}(X, T, \alpha)$-ergodic.

Proof. It is easily checked that $(X, \mathcal{B}, T, m, \alpha)$ is a Gibbs-Markov map in the sense of [2]. We have already seen that $\mathcal{E}(X, T, \alpha)=\mathfrak{T}\left(T, F^{\natural}\right) \cong \mathfrak{T}\left(T_{F^{\natural}}\right) \cap(X \times\{0\})^{2}$. By the construction of $F^{\natural},\left\{F^{\natural}(x)-F^{\natural}(y): x, y \in X\right\}$ generates $\mathbb{Z}_{0}^{\alpha \backslash\left\{a_{0}\right\}}$ and so $F^{\natural}: X \rightarrow \mathbb{Z}_{0}^{\alpha \backslash\left\{a_{0}\right\}}$ is aperiodic. It follows from the theorem in [2] that $T_{F^{\natural}}$ is exact, whence $\mathfrak{T}\left(T_{F^{\natural}}\right)$ is

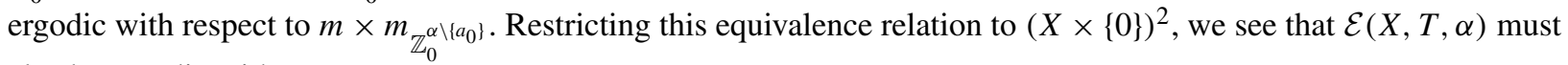
also be ergodic with respect to $m$.

Proposition 4.5. Suppose that $(X, T, \alpha)$ is a mixing $T M S$, that $\mathbb{G}$ is countable and that $\phi: X \rightarrow \mathbb{G}$ is aperiodic and has finite memory. Let $m \in \mathfrak{M}_{\alpha}(X)$ be a globally supported measure with the Markovian Gibbs property such that $(X, \mathcal{B}, m, T)$ is conservative and exact, then $m \times m_{\mathbb{G}}$ is $\mathfrak{T}(T)_{\widehat{\phi}}=\mathfrak{T}\left(T_{\phi}\right)$-ergodic. 
Proof. After possibly recoding, we may assume that $\phi(x)=\phi\left(x_{1}\right)$. Since $m$ is globally supported and Gibbs, $m$ is $\mathfrak{T}(T)$-nonsingular.

Let $A=\biguplus_{g \in \mathbb{G}} A_{g} \times\{g\} \in \mathcal{B}(X \times \mathbb{G})$ be $\mathfrak{T}\left(T_{\phi}\right)$-invariant. We claim first that $\forall s \in S, g \in \mathbb{G}$, either $s \subseteq A_{g} \bmod m$, or $m\left(s \cap A_{g}\right)=0$.

To see this, fix $s \in \alpha, g \in \mathbb{G}$ and let $\tilde{\alpha}_{s} \subset \mathcal{B}(s)$ be the first return time partition (to $s$ ), and $T_{s}: s \rightarrow s$ the induced map. The Markovian Gibbs property implies that $\left.m\right|_{s}$ is a $\left(s, T_{s}, \tilde{\alpha}\right)$-product measure. Since

$$
\mathcal{E}\left(s, T_{s}, \tilde{\alpha}\right) \subset \mathcal{E}(X, T, \alpha) \cap(s \times s) \subset \mathfrak{T}\left(T_{\phi}\right) \cap(s \times s),
$$

we have that $s \cap A_{g}$ is $\mathcal{E}\left(s, T_{s}, \tilde{\alpha}\right)$-invariant. By Lemma 4.4, either $s \subseteq A_{g} \bmod m$, or $m\left(s \cap A_{g}\right)=0$. If $m \times$ $m_{\mathbb{G}}(A)>0$, then $\exists g \in \mathbb{G}$ with $m\left(A_{g}\right)>0$, whence $\exists s \in S$ with $s \times\{g\} \subset A \bmod m \times m_{\mathbb{G}}$. Since $\phi$ is aperiodic, by Proposition 4.1, $\mathfrak{T}\left(T_{\phi}\right)$ is topologically transitive on $X \times \mathbb{G}$ and $A=X \times \mathbb{G} \bmod m \times m_{\mathbb{G}}$.

The following corollary was proved for Markov measures in [15].

Corollary 4.6. Suppose that $(X, T, \alpha)$ is a mixing TMS. Let $m \in \mathfrak{M}_{\alpha}(X)$ be a globally supported measure with the Markovian Gibbs property so that $(X, m, T, \alpha)$ is conservative and exact, then

(1) if $(X, T, \alpha)$ is almost onto, then $m$ is $\mathcal{E}(X, T, \alpha)$-ergodic;

(2) if not and $F^{\natural}=a+g-g \circ T+\overline{F^{\natural}}$ where $\overline{F^{\natural}}: X \rightarrow \mathbb{F}_{F^{\natural}}$ is aperiodic, then for each $b \in \mathbb{Z}_{0}^{S \backslash\left\{s_{0}\right\}}$ such that $m\left(\left[g \in b+\mathbb{F}_{F^{\natural}}\right]\right)>0,\left.m\right|_{\left[g \in b+\mathbb{F}_{F}\right]}$ is $\mathcal{E}(X, T, \alpha)$-non-singular and ergodic.

Proof. Fix $s_{0} \in S$ and apply Propositions 4.5, 1.0 and identity (0.2) to the aperiodic $F^{\natural}: X \rightarrow \mathbb{Z}_{0}^{S \backslash\left\{s_{0}\right\}}$ for (1), and to $\overline{F^{\natural}}: X \rightarrow \mathbb{F}_{F^{\natural}}$ for (2).

Of course this corollary applies to measures with $\alpha$-measurable derivatives.

\section{From exchangeable measures to conformal measures (TMS)}

Invariant recurrent measures for cocycle sub-relations. The following results are geared towards showing that recurrent, exchangeable, ergodic, locally finite measures have $\alpha$-measurable derivatives. Noting that $\mathcal{E}(X, T, \alpha)=$ $\mathfrak{T}\left(T, F^{\natural}\right)$, we consider the more general problem of identifying the ergodic invariant measures for $\mathfrak{T}(T, \phi)$ with $\alpha$ measurable $\phi$ 's, in the recurrent case. The reader is invited to recall the notation introduced in Eq. (1.0).

Theorem 5.0 (globally supported, aperiodic case). Suppose that $(X, T, \alpha)$ is a mixing TMS, $\mathbb{G}$ is countable and that $\phi: X \rightarrow \mathbb{G}$ is $\alpha$-measurable and aperiodic. If $v \in \mathfrak{M}(X)$ is globally supported, locally finite, $\mathfrak{T}(T, \phi)$-invariant, ergodic and recurrent, then $v \in \mathfrak{M}_{\alpha}(X)$ is Markov and

$$
\frac{\mathrm{d} \nu \circ T}{\mathrm{~d} \nu}=c \mathrm{e}^{h \circ \phi}, \quad \text { for some homomorphism } h: \mathbb{G} \rightarrow \mathbb{R} \text { and } c>0 .
$$

Proof. We divide the proof into several steps.

Step 1. $v \in \mathfrak{M}_{\alpha}(X)$, and $v$ is a $\mathfrak{T}(T)$-non-singular Markov measure.

To see this fix any $s \in S$ and let $\varphi_{s}(x):=\min \left\{n \geqslant 1: T^{n} x \in[s]\right\} \quad(x \in[s]), \tilde{\phi}:=\sum_{k=0}^{\varphi_{s}-1} \phi \circ T^{k}$, and $\tilde{\alpha}=$ $\left\{\left[s, a_{1}, \ldots, a_{n-1}, s\right]: n \in \mathbb{N}, \forall i a_{i} \in \alpha\right.$ and $\left.a_{i} \neq s\right\}$. Note that $\tilde{\alpha}$ is a partition of [s] modulo $v$ ( $v$ is recurrent). Also, $\tilde{\phi}$ is $\tilde{\alpha}$-measurable, and it is routine to check that

$$
\mathcal{E}\left([s], T_{[s]}, \tilde{\alpha}_{s}\right) \subseteq \mathfrak{G}\left(T_{[s]},\left(\tilde{\phi}, \varphi_{s}\right)\right)=\mathfrak{T}(T, \phi) \cap([s] \times[s]) .
$$

By Proposition 2.2, part 2, $\left.v\right|_{[s]}$ is recurrent with respect to $\left([s], T_{[s]}, \tilde{\alpha}_{s}\right)$ and thus, by Proposition 2.2, part 1, a mixture of ([s], $\left.T_{[s]}, \tilde{\alpha}_{s}\right)$-products.

If $n \geqslant 1$ and $\left[s, a_{1}, \ldots, a_{n}, t\right],\left[s, a_{1}^{\prime}, \ldots, a_{n}^{\prime}, t\right]$ are cylinders with $\sum_{j=1}^{n} \phi\left(a_{i}\right)=\sum_{j=1}^{n} \phi\left(a_{i}^{\prime}\right)$, then

$$
v\left(\left[s, a_{1}, \ldots, a_{n}, t\right] \cap T^{-(n+k)} b\right)=v\left(\left[s, a_{1}^{\prime}, \ldots, a_{n}^{\prime}, t\right] \cap T^{-(n+k)} b\right) \quad \forall k \geqslant 1 \text {, cylinder } b .
$$


It follows from this that each product measure component of $v_{[s]}$ (being a conditional probability with respect to the tail $\sigma$-algebra) is $\mathfrak{T}(T, \phi) \cap([s] \times[s])$-invariant (c.f. the proof of Lemma 12 of [8]). By $\mathfrak{T}(T, \phi) \cap([s] \times[s])$-ergodicity of $\left.\nu\right|_{[s]}$, there is actually only one component, whence $v([s])<\infty$.

To show the Markov property, we note that $\left.v\right|_{[s]}$, being exchangeable, is partially exchangeable on $[s]$ in the sense of [8]. By Proposition 15 of [8], it is the restriction to [s] of a Markov measure. The transition probability of $\left.v\right|_{[s]}$ does not depend on $s \in S$. Being globally supported and Markov, $v$ is $\mathfrak{T}(T)$-non-singular. Step 1 is established.

Step 2. Next, let $m \in \mathfrak{M}(X \times \mathbb{G})$ be the unique $\mathfrak{T}\left(T_{\phi}\right)$-invariant, ergodic measure satisfying $m(A \times\{0\})=v(A)$ $\forall A \in \mathcal{B}(X)$ (see Proposition 1.0). We claim that $m \ll v \times m_{\mathbb{G}}$ and that $m([s] \times\{g\})<\infty \forall s \in S, g \in \mathbb{G}$.

To prove $m \ll v \times m_{\mathbb{G}}$, assume $m(A \times\{g\})>0$. By ergodicity, $\exists A^{\prime} \subset A, m\left(A^{\prime} \times\{g\}\right)>0$ and $B \in \mathcal{B}(X), v(B)=$ $m(B \times\{0\})>0$ so that $A^{\prime} \times\{g\} \stackrel{\mathfrak{T}\left(T_{\phi}\right)}{\rightarrow} B \times\{0\}$, and in particular $A^{\prime} \stackrel{\mathfrak{T}(T)}{\rightarrow} B$. By $\mathfrak{T}(T)$-non-singularity of $v, v(A) \geqslant$ $v\left(A^{\prime}\right)>0$.

To show that $m([s] \times\{g\})<\infty \forall s \in S, g \in \mathbb{G}$, note that for each $z \in \mathbb{G}, m \circ Q_{z}$ is a $\sigma$-finite, $\mathfrak{T}\left(T_{\phi}\right)$-invariant, ergodic measure where $Q_{z}(x, y):=(x, y+z)$. Thus either $m \circ Q_{z} \propto m$, or $m \circ Q_{z} \perp m$. Let

$$
\mathbb{H}=\mathbb{H}(m):=\left\{z \in \mathbb{G}: m \circ Q_{z} \propto m\right\},
$$

then $\exists$ a homomorphism $h: \mathbb{H} \rightarrow \mathbb{R}$ such that $m \circ Q_{z}=\mathrm{e}^{-h(z)} m \forall z \in \mathbb{H}$. It follows from this, and $m \ll v \times m_{\mathbb{G}}$ that for $A \in \mathcal{B}(X)$,

$$
m(A \times\{z\})= \begin{cases}\mathrm{e}^{-h(z)} v(A), & z \in \mathbb{H}, \\ 0, & z \notin \mathbb{H},\end{cases}
$$

whence the local finiteness of $m$.

Step 3. $\mathbb{H}=\mathbb{G}$. Suppose that $g \in \mathbb{G}$ and fix $s \in S$. By aperiodicity, $\mathbb{F}_{\phi, s}=\mathbb{G}$ and $\exists n \geqslant 1, a=\overline{s a_{1} a_{2} \cdots a_{n-1}}$, $b=\overline{s b_{1} b_{2} \cdots b_{n-1}} \in \Pi_{n, s}$ satisfying $\phi_{n}(b)-\phi_{n}(a)=g$. Since $v$ is globally supported, $v\left(\left[s a_{1} a_{2} \cdots a_{n-1}\right]\right)$, $v\left(\left[s b_{1} b_{2} \cdots b_{n-1}\right]\right)>0$.

We claim that $\left[s a_{1} a_{2} \cdots a_{n-1}\right] \times\{0\} \stackrel{\mathfrak{T}\left(T_{\phi}\right)}{\rightarrow}\left[s b_{1} b_{2} \cdots b_{n-1}\right] \times\{g\}$ by the map $\left(\left(s, a_{1}, a_{2}, \ldots, a_{n-1}, x\right), z\right) \mapsto$ $\left(\left(s, b_{1}, b_{2}, \ldots, b_{n-1}, x\right), z+g\right)$. This is because

$$
\left(s, a_{1}, a_{2}, \ldots, a_{n-1}, x\right) \mapsto\left(s, b_{1}, b_{2}, \ldots, b_{n-1}, x\right)
$$

is a $\mathfrak{T}(T)$-holonomy $\left(\left[s a_{1} a_{2} \cdots a_{n-1}\right] \rightarrow\left[s b_{1} b_{2} \cdots b_{n-1}\right]\right)$ and $\phi_{n}\left(s, b_{1}, b_{2}, \ldots, b_{n-1}, x\right)-\phi_{n}\left(s, a_{1}, a_{2}, \ldots, a_{n-1}, x\right)$ $=\phi_{n}(b)-\phi_{n}(a)=g \forall x \in[s]$. Thus $m(X \times\{g\})>0$ and $g \in \mathbb{H}$.

Step 4. We now complete the proof of the theorem by showing that $\frac{\mathrm{d} v \circ T}{\mathrm{~d} \nu}=c \mathrm{e}^{h \circ \phi}$ for some $c>0$.

The proof of Step 2 shows that $m=\mathrm{e}^{-h} v \times \mathrm{d} m_{\mathbb{G}}$ (where $m_{\mathbb{G}}$ is the counting measure on $\mathbb{G}$ ). Since $m$ is by definition $\mathfrak{T}\left(T_{\phi}\right)$-invariant, for any $\mathfrak{T}(T)$-holonomy $K, \frac{\mathrm{d} v \circ K}{\mathrm{~d} v}(x)=\mathrm{e}^{h(\hat{\phi}(x, K x))}$. By Proposition $1.1, \frac{\mathrm{d} v \circ T}{\mathrm{~d} v}=c \mathrm{e}^{h \circ \phi}$ for some $c>0$.

Theorem 5.1 (openly supported, periodic case). Suppose that $\mathbb{G}$ is countable, that $(X, T, \alpha)$ is a mixing TMS, that $\mathbb{F}$ is a subgroup of $\mathbb{G}$ and that $\phi: X \rightarrow \mathbb{G}$ is $\alpha$-measurable and of form $\phi=a+g-g \circ T+\bar{\phi}$ where $a \in \mathbb{G}, g: X \rightarrow \mathbb{G}$ is $\alpha$-measurable and $\bar{\phi}: X \rightarrow \mathbb{F}$ is $\alpha$-measurable and aperiodic.

If $v \in \mathfrak{M}(X)$ is locally finite, has clopen support $U$, is recurrent and $\mathfrak{T}(T, \phi)$-invariant, ergodic, then $U=[g \in$ $\left.z_{0}+\mathbb{F}\right]$ for some $z_{0} \in \mathbb{G}$ and $\exists$ a homomorphism $h: \mathbb{G} \rightarrow \mathbb{R}, c>0$ and $\mu \in \mathfrak{M}_{\alpha}(X)$ Markov, such that $\frac{\mathrm{d} \mu \circ T}{\mathrm{~d} \mu}=c \mathrm{e}^{h \circ \phi}$, $v=\left.\mu\right|_{U}$.

Proof. As in the proof of Step 1 of Theorem 5.0, $v \in \mathfrak{M}_{\alpha}(X)$ is the restriction to $U$ of a global Markov measure and is thus $\mathfrak{T}(T) \cap(U \times U)$-non-singular.

Next, let $m \in \mathfrak{M}(X \times \mathbb{G})$ be the unique $\mathfrak{T}\left(T_{\phi}\right)$-invariant, ergodic measure satisfying $m(A \times\{0\})=v(A) \forall A \in \mathcal{B}(X)$ (see Proposition 1.0) and let $\bar{m}:=m \circ \pi$ where $\pi(x, y):=(x, y-g(x))$. Since $\pi^{-1} \circ T_{\phi} \circ \pi=T_{a+\bar{\phi}}$, we have that $\bar{m} \in \mathfrak{M}(X \times \mathbb{G})$ is $\mathfrak{T}\left(T_{\bar{\phi}}\right)$-invariant, ergodic. Also, $\mathbb{H}(m)=\mathbb{H}(\bar{m})$ (as defined in Step 2 of the proof of Theorem 5.0).

We claim first that $\mathbb{H}(\bar{m}) \subseteq \mathbb{F}$. To see this, assume w.l.o.g. that $m(X \times\{0\})>0$. If $A \in \mathcal{B}(X), g \in \mathbb{G}$ satisfy $m(A \times$ $\{g\})>0$, then by ergodicity, $\exists A^{\prime} \subset A, m\left(A^{\prime} \times\{g\}\right)>0$ and $B \in \mathcal{B}(X), m(B \times\{0\})>0$ so that $A^{\prime} \times\{g\} \stackrel{\mathfrak{T}\left(T_{\bar{\phi}}\right)}{\rightarrow} B \times\{0\}$. Since $\bar{\phi}: X \rightarrow \mathbb{F}$, it follows that $g \in \mathbb{F}$. 
Next, we claim that $\mathbb{H}(\bar{m}) \supseteq \mathbb{F}$. To see this, fix $s \in S,[s] \subset U$ and consider $m_{s}:=\left.m\right|_{[s] \times \mathbb{G}}$ which is $\mathfrak{T}\left(T_{\phi}\right) \cap$ $([s] \times \mathbb{G})^{2}$-invariant, ergodic. Now

$$
\mathfrak{T}\left(T_{\phi}\right) \cap([s] \times \mathbb{G})^{2}=\left(\mathfrak{T}(T)_{\hat{\phi}}\right) \cap([s] \times \mathbb{G})^{2}=(\mathfrak{T}(T) \cap([s] \times[s]))_{\hat{\phi}}
$$

and in a similar manner to Steps 2 and 3 of the proof of Theorem 5.0, we see that $\mathbb{H}(\bar{m})=\mathbb{F}$.

It follows that $\exists h: \mathbb{F} \rightarrow \mathbb{R}$ a homomorphism, $z_{0} \in \mathbb{G}$ and a $\sigma$-finite measure $\bar{v}$ on $X$ so that

$$
\bar{m}\left(A \times\left\{z+z_{0}\right\}\right)= \begin{cases}\mathrm{e}^{h(z)} \bar{v}(A), & z \in \mathbb{F}, \\ 0, & z \notin \mathbb{F} .\end{cases}
$$

Assume without loss of generality that $z_{0}=0$. Thus, for $K$ a $\mathfrak{T}(T)$-holonomy, $\frac{\mathrm{d} \bar{v} \circ K}{\mathrm{~d} \bar{v}}=\mathrm{e}^{-h(\hat{\bar{\phi}}(x, K x))}$, whence as in Step 4 of the of the proof of Theorem 5.0, $\frac{\mathrm{d} \bar{\nu} \circ T}{\mathrm{~d} \bar{\nu}}=c \mathrm{e}^{h \circ \bar{\phi}}$ for some $c>0$. Now

$$
\begin{aligned}
\nu(A) & =m(A \times\{0\})=\bar{m} \circ \pi^{-1}(A \times\{0\})=\bar{m}\left(\bigcup_{z \in \mathbb{G}} A \cap[g=z] \times\{z\}\right) \\
& =\sum_{z \in \mathbb{G}} \bar{m}(A \cap[g=z] \times\{z\})=\sum_{z \in \mathbb{F}} \mathrm{e}^{h(z)} \bar{v}(A \cap[g=z])=\int_{A \cap[g \in \mathbb{F}]} \mathrm{e}^{h \circ g} \mathrm{~d} \bar{\nu} .
\end{aligned}
$$

By Theorem 16.1 in [11], $\exists$ a homomorphism $H: \mathbb{G} \rightarrow \mathbb{R}$ with $\left.H\right|_{\mathbb{F}} \equiv h$. Fixing one such $H: \mathbb{G} \rightarrow \mathbb{R}$, and setting $\mathrm{d} \mu:=\mathrm{e}^{H \circ g} \mathrm{~d} \bar{\nu}$ we see that $\frac{\mathrm{d} \mu \circ T}{\mathrm{~d} \mu}(x)=c \mathrm{e}^{H(g(T x)-g(x))} \frac{\mathrm{d} \bar{\nu} \circ T}{\mathrm{~d} \bar{\nu}}=c \mathrm{e}^{H(g(T x)-g(x))-h(\bar{\phi}(x))}=c \mathrm{e}^{-H(\phi(x))}$.

Applications to exchangeability. Suppose that $(X, T, \alpha)$ is a TMS and that $v \in \mathcal{P}(X)$ is $\mathcal{E}(X, T, \alpha)$-invariant and ergodic, then for $s \in S N_{s}:=\sum_{n=0}^{\infty} 1_{[s]} \circ T^{n}$ is $\mathcal{E}(X, T, \alpha)$-invariant, whence constant $v$-a.e. Recall that $v$ is called $r e$ current, if $N_{s} \in\{0, \infty\}$ for all states $s$. We call $s \in S$ v-ephemeral if $1 \leqslant N_{s}<\infty v$-a.e., and the measure $v$ ephemeral if every state is $v$-ephemeral. Non-ephemerality is strictly weaker than recurrence.

We now apply the previous results to identify the non-ephemeral ergodic exchangeable measures on topological Markov shifts. For some (though not all) TMS there can be no other topologically $\sigma$-finite exchangeable measures, see Section 6 below.

We maintain the notation of the previous subsection. Let $(X, T, \alpha)$ be a mixing topological Markov shift, and suppose that $\mu \in \mathfrak{M}_{\alpha}(X)$ is globally supported and recurrent.

If $\frac{\mathrm{d} \mu \circ T}{\mathrm{~d} \mu}$ is $\alpha$-measurable, then by Proposition $2.0, \mu$ is exchangeable. We claim that its $\mathcal{E}(X)$-ergodic components are given by Proposition 4.6. To see that this proposition applies, we need to check that $\mu$ is Markovian, conservative, and exact: The Markov property is clear; The conservativity and exactness of recurrent Markov measures on mixing TMS are well-known (see e.g. [1]).

Thus the structure of exchangeable measures with $\alpha$-measurable derivative is understood. The following two results show that any locally finite, non-ephemeral, $\mathcal{E}(X)$-invariant, ergodic measure has a similar form, thus generalizing and clarifying Corollary 2.8 in [5]. We treat the recurrent case separately, because the result is easier to state in this case.

For $v \in \mathfrak{M}(X)$, let $S_{\infty}=S_{\infty}(v):=\left\{s \in S: v\left(\left[N_{s}=\infty\right]\right)>0\right\}$, where as before $N_{s}:=\sum_{n \geqslant 1} 1_{s} \circ T^{n}$.

Let $X$ be a (shift)-topologically transitive TMS, then (see e.g. [7]), $X=\biguplus_{k=1}^{N} X_{k}$ where $N \in \mathbb{N}$ and $X_{1}, \ldots, X_{N}$ are disjoint, clopen subsets of $X$ with $T X_{k}=X_{k+1} \bmod N$; and each $\left(X_{k}, T^{N}, \alpha_{N}\right)$ is mixing. This decomposition is called the periodic decomposition of $X, N=N_{X}$ is called the period of $X$ and each $X_{k}$ is called a basic, mixing set for $X$.

Theorem 5.2 (recurrent case). Suppose $(X, T, \alpha)$ is a TMS and $v \in \mathfrak{M}(X)$ is locally finite, recurrent and $\mathcal{E}(X, T, \alpha)$ invariant and ergodic; then there are

(1) $X^{\prime} \subset X \cap S_{\infty}^{\mathbb{N}}, \mathcal{E}(X, T, \alpha)$-invariant, such that $\left(X^{\prime}, T, \alpha\right)$ is a topologically transitive TMS and;

(2) a clopen, $\mathcal{E}(X, T, \alpha)$-invariant subset $U$ of a basic mixing set for $X^{\prime}$;

so that $v=\left.\mu\right|_{U}$ where $\mu \in \mathfrak{M}_{\alpha}\left(X^{\prime}\right)$ is Markov and $\frac{\mathrm{d} \mu \circ T^{N} X^{\prime}}{\mathrm{d} \mu}$ is $\alpha$-measurable. 
Proof. As in the proof of Step 1 of Theorem 5.0, $v$ is the restriction of a Markov measure to a union of initial states.

The associated stochastic matrix $p$ is recurrent (by assumption) and irreducible (as $v$ is $\mathcal{E}(X, T, \alpha)$-ergodic). Thus $X^{\prime}:=\left\{x \in S_{\infty}^{\mathbb{N}}: p_{x_{n}, x_{n+1}}>0 \forall n \geqslant 1\right\}$ is topologically transitive (with respect to the shift).

In case $\left(X^{\prime}, T, \alpha\right)$ is mixing, the result follows from Theorem 5.1.

In general $\left(X^{\prime}, T, \alpha\right)$ is transitive with periodic decomposition $X^{\prime}=\biguplus_{k=1}^{N} X_{k}^{\prime}$. Each $\left(X_{k}^{\prime}, T^{N}, \alpha_{N}\right)$ is mixing and $\mathfrak{T}(T)=\mathfrak{T}\left(T^{N}\right)$-invariant whence $v$ is supported on some $X_{k_{0}}^{\prime}$.

Thus, $v$ is $\mathcal{E}\left(X_{k_{0}}^{\prime}, T^{N}, \alpha_{N}\right)$-invariant, whence (by the mixing case) a mixture of conformal, Markov measures. However $v$ is $\mathfrak{T}\left(T^{N}\right)$-ergodic and so this mixture is trivial.

Remark. The TMS $X^{\prime}$ is not necessarily mixing, even if $X$ is mixing. To see this, let $X \subset\{0,1\}^{\mathbb{N}}$ be the (mixing) Fibonacci shift with transition matrix $\left(\begin{array}{ll}1 & 1 \\ 1 & 0\end{array}\right)$. The measure $v:=\delta_{(1010 \ldots)}$ is exchangeable, the corresponding TMS $X^{\prime}=\{(1010 \ldots),(0101 \ldots)\}$ being non-mixing (having period 2) with transition matrix $\left(\begin{array}{ll}0 & 1 \\ 1 & 0\end{array}\right)$.

Theorem 5.3 (non-ephemeral case). If $(X, T, \alpha)$ is a TMS and $v \in \mathfrak{M}(X)$ is locally finite, $\mathcal{E}(X, T, \alpha)$-invariant, ergodic and non-ephemeral, then there exist

(1) a cylinder $f=\left[f_{1}, \ldots, f_{K}\right] \subset \Sigma$ with $f_{1}, \ldots, f_{K} v$-ephemeral;

(2) $X^{\prime} \subset X \cap S_{\infty}^{\mathbb{N}}$ a $\mathcal{E}(X, T, \alpha)$-invariant $T M S$;

(3) a clopen, $\mathcal{E}(X, T, \alpha)$-invariant subset $U$ of a basic mixing set for $X^{\prime}$;

(3) a Markov measure $\mu \in \mathfrak{M}_{\alpha}\left(X^{\prime}\right)$

so that $\frac{\mathrm{d} \mu \circ T^{N_{X^{\prime}}}}{\mathrm{d} \mu}$ is $\alpha$-measurable and

$$
v=c \sum_{\sigma \in S_{K}, \sigma f \cap U \neq \emptyset} \delta_{\sigma f} \times\left.\mu\right|_{T^{K}(\sigma f) \cap U}
$$

where $\sigma f:=\left[f_{\sigma(1)}, \ldots, f_{\sigma(K)}\right]$.

Proof. Let $S_{e}$ be the set of $v$-ephemeral states and $S_{\infty}$ be the set of $v$-recurrent states. Let $\mathfrak{n}(x):=\min \{n \geqslant 1$ : $\left.x_{n} \in S_{\infty}\right\}$. We claim that $\mathfrak{n}$ is constant and that $\mathfrak{n}=\sum_{f \in S_{e}} N_{f}<\infty$.

To see this, we claim first that for $v$-a.e. $x, x_{k} \in S_{\infty} \forall k \geqslant \mathfrak{n}(x)$.

Since $S_{\infty} \neq \emptyset, \mathfrak{n}<\infty v$-a.e. Suppose that $x \in X, \mathfrak{n}(x)=K, x_{K}=s \in S_{\infty}$ and $v\left(\left[x_{1}, \ldots, x_{K}\right]\right)>0$. It follows that $v\left(\left[x_{1}, \ldots, x_{K}\right] \cap T^{-K}(\cdot)\right)$ is a locally finite, $\mathcal{E}\left([s], T_{[s]}, \tilde{\alpha}\right)$-invariant, $\mathfrak{T}\left([s], T_{[s]}, \tilde{\alpha}\right)$-ergodic measure on $[s]$ which is, by Proposition 2.2, a multiple of a $\left([s], T_{[s]}, \tilde{\alpha}\right)$-product measure. Thus

$$
x_{n} \in \begin{cases}S_{e}, & 1 \leqslant n \leqslant \mathfrak{n}(x), \\ S_{\infty}, & n>\mathfrak{n}(x),\end{cases}
$$

whence $\mathfrak{n}(x)=\sum_{f \in S_{e}} N_{f}(x)<\infty$, which is constant by $\mathcal{E}(X, T, \alpha)$-ergodicity. The result follows from this, and Theorem 5.2.

\section{Examples of ephemeral and non-ephemeral exchangeable measures}

Non-ephemeral exchangeable measures with ephemeral states. The following example is taken from [23]. Consider the TMS $X$ on the states $\{0,1,2,3\}^{\mathbb{N}}$ with transitions

$$
0 \leftrightarrow 1 \circlearrowleft 2 \rightarrow 3 \rightarrow 1
$$

and the exchangeable $v \in \mathcal{P}(X)$ given by

$$
v\left(\left[3, \epsilon_{1}, \epsilon_{2}, \ldots, \epsilon_{n}\right]\right):= \begin{cases}\frac{1}{2^{n}}, & \epsilon_{1}, \epsilon_{2}, \ldots, \epsilon_{n}=0,1, \\ 0 & \text { else. }\end{cases}
$$

TMS without locally finite ephemeral exchangeable measures. A TMS $(X, T, \alpha)$ with state space $S$ and transition matrix $A=\left(t_{i j}\right)_{i, j \in S \times S}$ has the finite images property, if $A$ has finitely many rows (equivalently $\{T a: a \in \alpha\}$ is finite). 
Examples include any TMS with finite state space, the full shift on a countable alphabet, and any shift obtained from the full shift by removing a finite collection of edges.

Proposition 6.0. If $(X, T, \alpha)$ has the finite images property and $v \in \mathfrak{M}(X)$ is topologically $\sigma$-finite, $\mathcal{E}(X, T, \alpha)$ invariant, ergodic, then $v$ is not ephemeral.

Proof. Let $v \in \mathfrak{M}(X)$ is topologically $\sigma$-finite, $\mathcal{E}(X, T, \alpha)$-invariant, ergodic. Suppose also that $v \in \mathfrak{M}(X)$ is ephemeral.

For $a \in S$ define $R(a), C(a): S \rightarrow\{0,1\}$ by $R(a)(b):=t_{a b}, C(a)(b):=t_{b a}$. The finite images property is that $\mathcal{R}:=\{R(a): a \in S\}$ is finite, say equal to $\left\{R_{1}, \ldots, R_{N}\right\}$. We claim that $\mathcal{C}:=\{C(a): a \in S\}$ is finite as well. To see this, note that

$$
S=\biguplus_{i=1}^{N} A_{i}, \quad \text { where } A_{i}=\left\{a \in S: R(a)=R_{i}\right\} .
$$

For each $i, C(a)$ is constant on $A_{i}$, because

$$
C(a)(b)=t_{b a}=R(b)(a)=R_{i}(a) \text { is independent of } b \in A_{i} .
$$

It follows that $|\{C(a): a \in S\}| \leqslant 2^{N}$.

Fix $(C, R) \in \mathcal{C} \times \mathcal{R}$ and let $S_{(C, R)}:=\{a \in S: C(a)=C, R(a)=R\}$. If $a \in S_{(C, R)}, x_{1}, x_{2}, \ldots, x_{n-1} \notin S_{(C, R)}$ and $\left[S_{(C, R)}\right] \cap T^{n}\left[a, x_{1}, \ldots, x_{n-1}\right] \neq \emptyset$ (where $\left[S_{(C, R)}\right]:=\bigcup_{s \in S_{(C, R)}}[s]$ ), then $\exists b \in S_{(C, R)}$ with $t_{x_{n-1}, b}=1$ whence $C\left(x_{n-1}\right)=1$ and $T^{n}\left[a, x_{1}, \ldots, x_{n-1}\right] \supset\left[S_{(C, R)}\right]$.

It follows that $\left(A_{(C, R)}, T_{A_{(C, R)}}, \alpha_{(C, R)}\right)$ is a full fibred system where $A_{(C, R)}:=\left\{x \in\left[S_{(C, R)}\right]: x_{n} \in S_{(C, R)}\right.$ infinitely often\} and

$$
\alpha_{(C, R)}:=\left\{\left[a, x_{1}, \ldots, x_{n-1}\right]: n \geqslant 1, a \in S_{(C, R)}, x_{k} \notin S_{(C, R)}, \sum_{b \in S_{(C, R)}} t_{x_{n-1}, b}>0\right\} .
$$

Also $\mathcal{E}\left(A_{(C, R)}, T_{A_{(C, R)}}, \alpha_{(C, R)}\right) \subset \mathcal{E}(X, T, \alpha) \cap A_{(C, R)} \times A_{(C, R)}$.

Since $\mathcal{C} \times \mathcal{R}$ is finite, $\exists N_{0} \geqslant 1,(C, R) \in \mathcal{C} \times \mathcal{R}$ such that $v\left(T^{-N_{0}} A_{(C, R)}\right)>0$. By topological $\sigma$-finiteness, $\exists N \geqslant N_{0}, a \in \alpha_{N}$ such that $0<v\left(a \cap T^{-N} A_{(C, R)}\right)<\infty$.

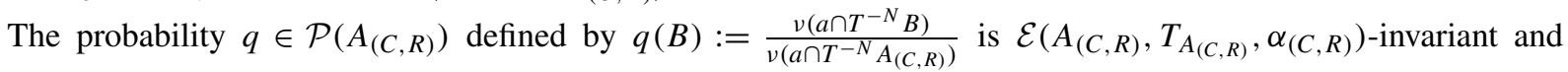

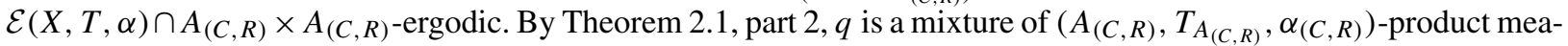
sures, but by $\mathcal{E}(X, T, \alpha) \cap A_{(C, R)} \times A_{(C, R)}$-ergodicity, there is only one component and $q$ is a $\left(A_{(C, R)}, T_{A_{(C, R)}}, \alpha_{(C, R)}\right)$ product measure.

In particular $q$ is recurrent with respect to $\left(A_{(C, R)}, T_{A_{(C, R)}}, \alpha_{(C, R)}\right)$ and $v$ cannot be ephemeral.

TMS with recurrent and ephemeral locally finite exchangeable measures. Consider the simple, aperiodic random walks TMS's $X_{d}$ for $d \in \mathbb{N}$ where:

$$
X_{d}:=\left\{x \in\left(\mathbb{Z}^{d}\right)^{\mathbb{N}}:\left\|x_{n+1}-x_{n}\right\|_{\infty} \leqslant 1 \forall n \geqslant 1\right\}
$$

equipped with the shift map $T$ and partition $\alpha:=\left\{[s]: s \in \mathbb{Z}^{d}\right\}$. Note that

(1) $\left(X_{d}, T, \alpha\right)$ is almost onto, so by Proposition 4.6, any globally supported, Markov measure on $X$ is $\mathcal{E}(X, T, \alpha)$ ergodic;

(2) $X_{d}=X^{d}$ where $X:=X_{1}$.

Now let $\mu \in \mathfrak{M}_{\alpha}(X)$ be a recurrent, $\mathcal{E}(X, T, \alpha)$-invariant, ergodic, measure on $X$. By Theorem 4.3, $\mu$ is Markov with $\alpha$-measurable derivative $\frac{\mathrm{d} \mu \circ T}{\mathrm{~d} \mu}$.

The symmetric, global, random walk measure $m$ on $X$ given by 


$$
m\left(\left[a_{1}, a_{2}, \ldots, a_{n}\right]\right)= \begin{cases}\frac{1}{3^{n}}, & \left|a_{k+1}-a_{k}\right| \leqslant 1,1 \leqslant k \leqslant n-1, \\ 0 & \text { else }\end{cases}
$$

is null recurrent and of this form. Note however that $m_{d}:=\underbrace{m \times \cdots \times m}_{d \text {-times }}$ is transient on $X_{d} \forall d \geqslant 3$.

Proposition 6.1. For each $d \geqslant 1$ there is a positively recurrent, exchangeable, Markov measure $\mu \in \mathfrak{M}_{\alpha}\left(X_{d}\right)$.

Proof. It suffices to find a positively recurrent, exchangeable, Markov measure $\mu \in \mathfrak{M}_{\alpha}(X)$ for then $\mu \underbrace{\times \cdots \times}_{d \text {-times }} \mu \in$ $\mathfrak{M}_{\alpha}\left(X_{d}\right)$ is as required.

To this end, fix $0<z<1$, set $\pi_{s}=z^{|s|}$ and let $\mu \in \mathfrak{M}_{\alpha}(X)$ be the Markov measure with $\mu([s])=\pi_{s}$ and $\frac{\mathrm{d} \mu \circ T}{\mathrm{~d} \mu}$ $\alpha$-measurable (as in Proposition 3.0). The underlying stochastic matrix is given by for $s \in \mathbb{Z}, i=0, \pm 1$ :

$$
p_{s, s+i}= \begin{cases}\frac{z^{i}}{z^{-1}+1+z}, & s \in \mathbb{Z}, s>0, i=0, \pm 1, \\ \frac{z^{-i}}{z^{-1}+1+z}, & s \in \mathbb{Z}, s<0, i=0, \pm 1, \\ \frac{z^{|i|}}{2 z+1}, & s=0, i=0, \pm 1 .\end{cases}
$$

An invariant distribution on $\mathbb{Z}$ for $P$ is given by

$$
c_{t}= \begin{cases}\frac{1}{1+z+z^{2}}, & t=0, \\ \frac{z^{2|t|-1}}{2 z+1}, & t \neq 0 .\end{cases}
$$

Since $\sum_{t \in S} c_{t}<\infty$, the stochastic matrix $P$ is positively recurrent.

Proposition 6.2. Set $Z:=\left\{x \in X: N_{s}(x)=0 \forall s \leqslant 0, N_{s}(x)=2 \forall s \geqslant 1\right\}$. There is a unique $\mathcal{E}(X, T, \alpha)$-invariant, ergodic measure $\bar{v} \in \mathfrak{M}_{\alpha}(X)$ which is carried by $Z$ and such that $\bar{v}([1])=1$. This measure is non-atomic and ephemeral.

Proof. Set $\mathcal{E}:=\mathcal{E}(X, T, \alpha), \Omega=\{0,1\}^{\mathbb{N}}, S: \Omega \rightarrow \Omega$ the left shift, and define $\vartheta: \Omega \rightarrow\{1,2\}^{\mathbb{N}}$ by $\vartheta(x):=x_{1}$.

Step 1 . Construction of a homeomorphism $\Phi: Z \cap[1] \rightarrow \Omega:=\{1,2\}^{\mathbb{N}}$ which carries $\mathcal{E} \cap(Z \cap[1])^{2}$ onto $\mathfrak{G}(S, \vartheta) \cap$ $(\Omega \times \Omega)$.

Call $K \in \mathbb{N}$ good for $x \in Z \cap[1]$ if $\#\left\{1 \leqslant k \leqslant 2 K: x_{k}=s\right\}=2 \forall 1 \leqslant s \leqslant K$ and let $\kappa(x):=\min \{K \geqslant 1$ : $K \operatorname{good}$ for $x$ \}. The possibilities for $\kappa(x)$ are:

(1) $\kappa(x)=1$ in case $x=(1,1, \tau(x)+(1,1,1, \ldots))$ where $\tau(x) \in Z \cap[1]$, and

(2) $\kappa(x)=2$ in case $x=(1,2,1,2, \tau(x)+(2,2,2, \ldots))$ where $\tau(x) \in Z \cap[1]$.

Note that $\tau: Z \cap[1] \rightarrow Z \cap[1]$ and $\kappa$ are related by

$$
\tau(x):=T^{2 \kappa(x)}(x)-(\kappa(x), \kappa(x), \ldots) .
$$

It follows that

$$
\begin{aligned}
& (x, y) \in \mathcal{E}(X, T, \alpha) \cap(Z \cap[1] \times Z \cap[1]) \\
& \quad \Leftrightarrow \quad \exists k, \ell \geqslant 1 \ni \kappa\left(\tau^{j+k} x\right)=\kappa\left(\tau^{j+\ell} y\right) \forall j \geqslant 1, \& \sum_{j=0}^{k} \kappa\left(\tau^{j} x\right)=\sum_{j=0}^{\ell} \kappa\left(\tau^{j} y\right) .
\end{aligned}
$$

Defining $\Phi: Z \cap[1] \rightarrow \Omega:=\{1,2\}^{\mathbb{Z}_{+}}$by $\Phi(x)_{n}:=\kappa\left(\tau^{n} x\right)$, we have that

$$
\Phi \times \Phi(\mathcal{E}(X, T, \alpha) \cap(Z \cap[1] \times Z \cap[1]))=\mathfrak{G}(S, \vartheta) \cap(\Phi(Z \cap[1]) \times \Phi(Z \cap[1]))
$$

where $S$ is the shift map on $\Omega$ and $\vartheta(x):=x_{1}$. 
Evidently $\Phi: Z \cap[1] \rightarrow \Phi(Z \cap[1])$ is a homeomorphism. We claim next that $\Phi(Z \cap[1])=\Omega$. To see this set $a(1):=(1,1), a(2):=(1,2,1,2)$ and define $\pi: \Omega \rightarrow \mathbb{Z}^{\mathbb{N}}$ by

$$
\pi(\omega):=\left(a\left(\omega_{0}\right)+s_{0}, a\left(\omega_{1}\right)+s_{1}, \ldots, a\left(\omega_{n}\right)+s_{n}, \ldots\right)
$$

where $s_{n}=s_{n}(\omega)$ is defined by $s_{0}=0, s_{n+1}=s_{n}+\omega_{n}$. Evidently $\pi \circ \Phi=\left.\operatorname{Id}\right|_{Z \cap[1]}$, whence $\pi(\Omega)=Z \cap[1]$.

Step 2. There is a unique $\mathfrak{G}(S, \vartheta)$-invariant $\mu \in \mathcal{P}(\Omega)$.

It is not hard to check that if $p(\omega)=p^{\omega}(\omega=1,2)$ where $p+p^{2}=1\left(p=\frac{\sqrt{5}-1}{2}\right)$, and $\mu \in \mathcal{P}(\Omega)$, $\mu\left(\left[\omega_{1}, \ldots, \omega_{n}\right]\right)=\prod_{k=1}^{n} p\left(\omega_{k}\right)=p^{\vartheta_{n}(\omega)}$, then $\mu$ is $\mathfrak{G}(S, \vartheta)$-invariant. To see uniqueness of this $\mu$, we note first that

$$
\mathcal{E}(\Omega) \subset \mathfrak{G}(S, \vartheta) \subset \mathfrak{G}(S) .
$$

If $\bar{\mu} \in \mathcal{P}(\Omega)$ is $\mathfrak{G}(S, \vartheta)$-invariant, ergodic, then it is $\mathcal{E}(\Omega)$-invariant, whence by de-Finetti an average of product measures; and $\mathfrak{G}(S)$-ergodic, whence a product measure. Writing $\bar{\mu}=\prod q$, we have by $\mathfrak{G}(S, \vartheta)$-invariance that $q(1)^{2}=\mu([11])=\mu([2])=q(2)$ whence $\bar{\mu}=\mu$.

Step 3. There is a unique $\mathcal{E} \cap(Z \cap[1])^{2}$ ergodic probability measure $v \in \mathcal{P}(Z \cap[1])$ and this measure is non-atomic.

This follows from Steps 1 and 2: Any $P \in \mathcal{P}(\Omega)$ is $\mathfrak{T}(\Omega, S)$-invariant, ergodic iff $P \circ \Phi \in \mathcal{P}(Z \cap[1])$ is $\mathcal{E} \cap(Z \cap$ $[1])^{2}$-invariant, ergodic. The required probability is $v:=\mu \circ \Phi$ where $\mu$ is as in Step 2.

To complete the proof of the theorem, we take for $\bar{v}$ the unique $\mathcal{E}$-invariant, ergodic measure $\bar{v}$ on $Z$ so that $\left.\bar{v}\right|_{Z \cap[1]}=v$. If $\lambda$ is another $\mathcal{E}$-invariant, ergodic measure on $Z$ so that $\lambda(Z \cap[1])=1$, then by Step $3,\left.\lambda\right|_{Z \cap[1]}=v$, whence $\lambda=\bar{v}$.

Finally, we note that $Z=\bigcup_{k=1}^{\infty}\left\{\left(k, k-1, \ldots, 2,1,1,2, \ldots, k-1, k, u^{k}(x)\right): x \in Z \cap[1]\right\}$ where $u(x)_{n}:=x_{n}+1$, whence $\bar{v} \in \mathfrak{M}_{\alpha}(X)$. In particular, $\bar{v}$ is locally finite.

Remark. It follows from Theorem 2.2 in [4] that the only partition-bounded, $\mathfrak{T}(T)$-invariant, ergodic measures on $X$ are the random walk measures $m_{f}$ of form

$$
m_{f}\left(\left[s_{1}, s_{2}, \ldots, s_{n}\right]\right)=\pi_{s_{1}} p_{s_{1}, s_{2}} \ldots p_{s_{n-1}, s_{n}}
$$

with $p_{s, s+\epsilon}:=f_{\epsilon}(\epsilon= \pm 1)$, where $f_{\epsilon}=z^{\epsilon} p$ for some $0<p \leqslant \frac{1}{3}, z=z_{ \pm}(p)=\frac{1}{2}\left(\frac{1}{p}-1 \pm \sqrt{\left(\frac{1}{p}-1\right)^{2}-4}\right)$ and $\pi_{s}=z^{s}(s \in \mathbb{Z})$. The only recurrent measure of this form is the symmetric one with $p=\frac{1}{3}, z=1$. All the others are ephemeral and not $\mathcal{E}(X, T, \alpha)$-ergodic. Their ergodic decompositions may be of interest.

\section{Part 3. Exchangeability for $\beta$-expansions}

\section{7. $\beta$-expansions}

Definition of $\beta$-expansions. Fix a non-integer $\beta>1$ and let $I:=[0,1]$. The $\beta$-transformation is $T=T_{\beta}: I \rightarrow I$, where $T x:=\{\beta x\}$. The $\beta$-expansion of $x \in I$ is $\pi(x)=\pi_{\beta}(x)=\left(\epsilon_{1}, \epsilon_{2}, \ldots\right) \in\{0,1, \ldots,[\beta]\}^{\mathbb{N}}$ where $\epsilon_{n}:=$ $\left[\beta T_{\beta}^{n-1} x\right]$. Evidently

$$
x=\sum_{n=1}^{\infty} \frac{\epsilon_{n}}{\beta^{n}} .
$$

This should be thought of as an expansion to a 'non-integer base' (of course, if $\beta \in \mathbb{N} \backslash\{1\}$, this is just the expansion to the base $\beta$ ). The $\beta$-expansions were introduced by Rényi [24], who together with Parry [21] also initiated the study of their stochastic behaviour (when $x \in[0,1]$ is distributed according to the absolutely continuous invariant probability measure of $T_{\beta}$ ).

For $\beta$ an integer the collection of $\beta$-expansions is a full-shift on $\beta=[\beta]$ symbols, and $\epsilon_{n}$ are i.i.d.'s. For general $\beta$ 's the collection of $\beta$-expansions is not a topological Markov shift (see below), so the digit process $\left\{\epsilon_{n}\right\}$ is not even Markov.

Nevertheless, various authors have shown that the digit process shares many of the properties of an i.i.d. process: SLLN, Kolmogorov and Hewitt-Savage zero-one laws, CLT, LLT (see [24,21,3]). We shall contribute to this list, by establishing a suitable version of the de-Finetti theorem. 
Basic properties of the $\beta$-transformation. For the basic results see [12,21,24]. Here we mention only a few that are used in the sequel. The $\beta$-transformation leaves Lebesgue measure $m$ quasi invariant $\left(m \circ T^{-1} \sim m\right.$ ). It is known that $(I, \mathcal{B}, m, T)$ is an exact endomorphism with a Lebesgue-equivalent invariant probability. The triple $(I, T, \alpha)$ where $\alpha:=\left\{\left[\frac{j}{\beta}, \frac{j+1}{\beta}\right)\right\}_{j=0}^{[\beta]-1} \cup\left\{\left[\frac{[\beta]}{\beta}, 1\right)\right\}$ is a fibred system.

The $\beta$-shift is the closure of the collection of $\beta$-expansions: $X_{\beta}:=\overline{\pi_{\beta}(I)}$. Set

$$
\omega=\omega_{\beta}:= \begin{cases}\left(\overline{\eta_{1}, \eta_{2}, \ldots, \eta_{q-1}, \eta_{q}-1}\right), & \pi_{\beta}(1)=\left(\eta_{1}, \eta_{2}, \ldots, \eta_{q-1}, \eta_{q}, \overline{0}\right), \\ \pi_{\beta}(1) & \text { else. }\end{cases}
$$

The following is in [21]:

$$
X_{\beta}=\left\{y \in\{0,1, \ldots,[\beta]\}^{\mathbb{N}}: y_{k}^{\infty} \prec \omega \forall k \geqslant 1\right\}
$$

where $x \prec y$ means $\exists n \geqslant 1, x_{n}<y_{n}, x_{1}^{n-1}=y_{1}^{n-1}$.

Full cylinders. A cylinder $\left[x_{1}, \ldots, x_{N}\right]$ with $T^{N}\left[x_{1}, \ldots, x_{N}\right]=X_{\beta}$ is called full. Full cylinders were considered in $[6,17,21,24,28,30]$.

Not every cylinder is full. The image of a general cylinder $a=\left[a_{1}, \ldots, a_{N}\right] \in \alpha_{N}$ is given by

$$
T^{N}\left[a_{1}, \ldots, a_{N}\right]=\left\{y \in X_{\beta}: y \prec \omega_{K_{N}(a)+1}^{\infty}\right\},
$$

where

$$
K_{N}(a):= \begin{cases}0, & \nexists 1 \leqslant n \leqslant N, a_{N-n+1}^{N}=\omega_{1}^{n}, \\ \max \left\{1 \leqslant n \leqslant N, a_{N-n+1}^{N}=\omega_{1}^{n}\right\} & \text { else. }\end{cases}
$$

By (7.1), if $K_{N}(a)=0$, then $a$ is full.

It is standard to check, noting that $\omega \in X_{\beta}$ and using (7.1), that if $\nexists n$ s.t. $x_{n}^{\infty}=\omega$, then $K_{N}\left(x_{1}, \ldots, x_{N}\right)=0$ infinitely often.

Set $\Gamma:=\left\{x \in X_{\beta}: \exists n \geqslant 1, x_{n}^{\infty}=\omega\right\}$, then $\pi: I \backslash \pi^{-1} \Gamma \rightarrow X_{\beta} \backslash \Gamma$ is invertible and $\pi \circ T_{\beta}=T \circ \pi$ where $T: X_{\beta} \rightarrow X_{\beta}$ is the shift. Accordingly set

$$
X_{\beta, 0}:=X_{\beta} \backslash \Gamma \text {. }
$$

The previous paragraph says that any $x \in X_{\beta, 0}$ belongs to infinitely many full cylinders.

This allows us to make the following definitions:

$$
\begin{aligned}
& \psi: X_{\beta} \backslash \Gamma \rightarrow \mathbb{N}, \quad \psi(x):=\min \left\{N \geqslant 1, T^{N}\left[x_{1}, \ldots, x_{N}\right]=X_{\beta}\right\}, \\
& S: X_{\beta, 0} \rightarrow X_{\beta, 0}, \quad S(x):=T^{\psi(x)}(x) .
\end{aligned}
$$

We call $S$ a Bernoulli jump transformation (see page 133 of [27]). Evidently $\left(X_{\beta, 0}, S, \tilde{\alpha}\right)$ is a full fibred system where

$$
\tilde{\alpha}:=\left\{a \in \alpha_{n}: n \geqslant 1, a \subset[\psi=n]\right\} .
$$

We have

$$
\mathcal{E}\left(X_{\beta, 0}, S, \tilde{\alpha}\right) \subset \mathfrak{T}\left(\left.T\right|_{X_{\beta, 0}}\right) \subset \mathfrak{T}(S) \subset \mathfrak{G}\left(\left.T\right|_{X_{\beta, 0}}\right) .
$$

Ergodic properties of the tail and exchangeable relations of $X_{\beta}$. We abuse notation and denote the Lebesgue measure on $I$ and the measure it induces on $X_{\beta}$ by the same symbol $m$.

Proposition 7.0. For every $\beta>1, m$ is $\mathcal{E}(I, T, \alpha)$-ergodic and invariant.

Proof. Viewing $T$ on $I$, we see that $\frac{\mathrm{d} m \circ T}{\mathrm{~d} m}=T^{\prime}$ is $\alpha$-measurable, so $m$ is $\mathcal{E}(I, T, \alpha)$-invariant by Proposition 2.0.

Viewing $S$ on $I$, we see that it is a piecewise onto affine map, and so $m$ is a $(S, \tilde{\alpha})$-product measure. Thus, by Theorem 2.1, $\left(\mathcal{I}\left(X_{\beta}, S, \tilde{\alpha}\right)\right) \stackrel{m}{=}\left\{\emptyset, X_{\beta}\right\}$. By (7.3) $\mathfrak{I}\left(\mathcal{E}\left(X_{\beta}, T, \alpha\right)\right) \subseteq \mathfrak{I}\left(\mathcal{E}\left(X_{\beta}, S, \tilde{\alpha}\right)\right) \stackrel{m}{=}\left\{\emptyset, X_{\beta}\right\}$.

Proposition 7.1. $\mathfrak{T}(T)$ is uniquely ergodic: If $\mu \in \mathfrak{M}\left(X_{\beta}\right)$ is topologically $\sigma$-finite and $\mathfrak{T}(T)$-invariant, then $\mu=c m$ for some $c>0$. 
Proof. Let $\mu \in \mathfrak{M}\left(X_{\beta}\right)$ be topologically $\sigma$-finite and $\mathfrak{T}(T)$-ergodic, invariant. It is easy to check that $\mathfrak{T}(T)$ equivalence classes are dense, therefore by local finiteness $\mu$ must be non-atomic. In particular $\mu\left(X_{\beta} \backslash X_{\beta, 0}\right)=0$, so we may work on $X_{\beta, 0}$.

We claim that $\mu$ is locally finite on $X_{\beta, 0}$. To see this, note first that by topological $\sigma$-finiteness, there is a cylinder set of positive, finite measure. By non-atomicity, we may assume that the cylinder is full. Namely: $\exists b \in \alpha_{N} \cap \tilde{\alpha}_{N^{\prime}}$ with $0<\mu(b)<\infty$. Now let $x \in X$. Since $T^{N} b=X$, we may define the $\mathfrak{T}(T)$-holonomy $\kappa: x_{1}^{N} \rightarrow b$ by $\kappa\left(x_{1}^{N}, z\right):=(b, z)$. By $\mathfrak{T}(T)$-invariance,

$$
\mu\left(\left[x_{1}^{N}\right]\right) \leqslant \mu\left(\kappa\left[x_{1}^{N}\right]\right) \leqslant \mu(b)<\infty
$$

and $\mu$ is locally finite on $X_{\beta, 0}$.

By (7.3), $\mu$ is $\mathfrak{T}(S)$-ergodic and $\mathcal{E}\left(X_{\beta, 0}, S, \tilde{\alpha}\right)$-invariant. By Proposition 2.2, $\mu$ is a $(S, \tilde{\alpha})$-product measure.

The $\mathfrak{T}(T)$-invariance implies that $\exists t>0$ so that $\mu(a)=t^{n} \forall a \in \tilde{\alpha} \cap \alpha_{n}$. To see this, suppose that $a \in \tilde{\alpha} \cap \alpha_{k}$, $b \in \tilde{\alpha} \cap \alpha_{\ell}$, then

$$
[\underbrace{a, \ldots, a}_{\ell \text {-times }}] \stackrel{\mathfrak{T}(T)}{\longrightarrow}[\underbrace{b, \ldots, b}_{k \text {-times }}] \text { by }(\underbrace{a, \ldots, a}_{\ell \text {-times }}, x) \mapsto(\underbrace{b, \ldots, b}_{k \text {-times }}, x)
$$

so $\mu(a)^{\ell}=\mu(b)^{k}$. The case $k=\ell$ shows that $\exists t_{k}>0$ so that $\mu(a)=t_{k} \forall a \in \tilde{\alpha} \cap \alpha_{k}$ and the other cases show that $\exists t>0$ so that $t_{k}^{1 / k}=t \forall k \geqslant 1, \tilde{\alpha} \cap \alpha_{k} \neq \emptyset$. This $t>0$ is uniquely determined by $\sum_{a \in \tilde{\alpha}} \mu(a)=1$, whence $t=\frac{1}{\beta}$ and $m=\mu$.

Note that the proof of Proposition 7.1 only used the existence of one cylinder with positive, finite $\mu$-measure.

As before, define $F^{\natural}: X_{\beta} \rightarrow \mathbb{Z}_{0}^{[\beta]}=\mathbb{Z}^{[\beta]}$ by $F^{\natural}(x)_{j}:=\delta_{j, x_{1}}$. (Here and throughout [-] is the largest integer lower bound, i.e. $[\beta]<\beta$.) Write $x_{1}^{N} \bowtie y_{1}^{N}$ if $F_{N}^{\natural}(x)=F_{N}^{\natural}(y)$ and $\exists k, \ell,\left[x_{1}^{N}\right] \in \tilde{\alpha}_{k},\left[y_{1}^{N}\right] \in \tilde{\alpha}_{\ell}$.

Lemma 7.2. $\mathcal{E}\left(X_{\beta, 0}, T, \alpha\right)=\left\{(x, y) \in\left(X_{\beta, 0}\right)^{2}: x_{N+1}^{\infty}=y_{N+1}^{\infty}\right.$ and $x_{1}^{N} \bowtie y_{1}^{N}$ for some $\left.N\right\}$.

\section{Conformal measures: existence and ergodicity}

Restricted conformal measures. Fix $J \subset\{0,1, \ldots,[\beta]\},|J|>1$. Note that $X_{\beta, 0}(J):=X_{\beta, 0} \cap J^{\mathbb{N}} \neq \emptyset$, because $j_{1}<j_{2} \in J \Rightarrow\left(j_{1}, j_{1}, \ldots\right) \in X_{\beta, 0}(J)$.

Setting $\alpha_{n}(J):=\left\{[\underline{a}] \in \alpha_{n}: \underline{a} \in J^{n}\right\}$ and $\tilde{\alpha}(J):=\bigcup_{n=1}^{\infty} \tilde{\alpha} \cap \alpha_{n}(J)$, we have that $\left(X_{\beta, 0}(J), S, \tilde{\alpha}(J)\right)$ is a full fibred system, whence $X_{\beta, 0}(J)$ is either a singleton, or uncountable. In the latter case $X_{\beta}(J):=X_{\beta} \cap J^{\mathbb{N}}=\bar{X}_{\beta, 0}(J)$. ${ }^{5}$

As before, we have

$$
\mathcal{E}\left(X_{\beta, 0}(J), S, \tilde{\alpha}(J)\right) \subset \mathfrak{T}\left(\left.T\right|_{X_{\beta, 0}(J)}\right) \subset \mathfrak{T}\left(\left.S\right|_{X_{\beta, 0}(J)}\right) \subset \mathfrak{G}\left(\left.T\right|_{X_{\beta, 0}(J)}\right) .
$$

Thus, in the infinite case, $\mathfrak{G}(T) \cap\left[X_{\beta, 0}(J) \times X_{\beta, 0}(J)\right]$ is minimal: Any equivalence class is dense.

Proposition 8.0. Suppose that $X_{\beta}(J)$ is infinite, and let $H: J \rightarrow \mathbb{R}_{+}$, then

(1) there is a unique $\lambda>0$ and $\left(\frac{1}{\lambda H}, T\right)$-conformal $\mu_{J, H} \in \mathcal{P}\left(X_{\beta}(J)\right)$;

(2) $\mu_{J, H}$ is a $(S, \tilde{\alpha})$-product measure and is $\mathcal{E}\left(X_{\beta}\right)$-invariant and ergodic.

Proof. In case $\omega_{\beta}$ is eventually periodic, $X_{\beta}(J)$ is sofic (see [6]) and a continuous, equivariant image of a TMS. Existence follows from Ruelle's Perron-Frobenius theorem, which provides a non-atomic, $\left(\frac{1}{\lambda H}, T\right)$-conformal measure.

In the case that $\omega_{\beta}$ is not eventually periodic, we prove existence (as in Sections 2 and 3 of [30]) as follows: Endow $X_{\beta}(J)$ with the lexicographic order topology, disconnecting it at $\Gamma:=\bigcup_{n \in \mathbb{Z}} T^{n}\left\{(\overline{0}), \omega_{\beta}\right\}$ to obtain the compact metric space

$$
Y=Y_{\beta}(J):=\left(X_{\beta}(J) \backslash \Gamma\right) \cup(\Gamma \times\{-,+\}) .
$$

5 The singleton case is possible: If $\beta=\frac{3+\sqrt{5}}{2}$, then $\omega=(2, \overline{1})$ and $X_{\beta, 0}(\{1,2\})=\{(1,1, \ldots)\}$. 
There are continuous maps $T_{Y}: Y \rightarrow Y$ and $\pi: Y \rightarrow X_{\beta}(J)$ defined by

$$
\left.\left(T_{Y}\right)\right|_{X_{\beta}(J) \backslash \Gamma} \equiv T,\left.\quad \pi\right|_{X_{\beta}(J) \backslash \Gamma} \equiv \mathrm{Id}, \quad T_{Y}(\gamma, \epsilon):=(T \gamma, \epsilon), \quad \pi(\gamma, \epsilon):=\gamma,
$$

so that $\pi \circ T_{Y}=T \circ \pi$. It follows that $H \circ \pi: Y \rightarrow \mathbb{R}_{+}$is continuous. By Schauder's fixed point theorem, $\exists v_{Y} \in \mathcal{P}(Y)$ $\left(\frac{1}{\lambda H \circ \pi}, T_{Y}\right)$-conformal for some $\lambda>0$.

In order to show that $\nu_{Y}$ descends to a conformal measure on $X_{\beta}(J)$, it is sufficient to show that $v_{Y}\left(\pi^{-1} \Gamma\right)=0$, because in this case $v_{Y}$ is supported on $X_{\beta}(J) \backslash \Gamma$ so $\pi:\left(Y, \mathcal{B}(Y), v_{Y}\right) \rightarrow\left(X_{\beta}(J), \mathcal{B}, v_{Y} \circ \pi^{-1}\right)$ is a measure theoretic isomorphism. The measure $\mu_{J, H}:=v_{Y} \circ \pi^{-1}$ is then the required conformal measure on $X_{\beta, 0}$.

Step 1. $v_{Y}\{((\overline{0}), \pm)\}=0$. Otherwise one of the preimages $z=((j, \overline{0}), \pm)$ has positive measure. The exchangeable orbit of $((j, \overline{0}), \pm)$ is $\{((\underbrace{0, \ldots, 0}_{n \text {-times }}, j, \overline{0}), \pm)\}_{n=1}^{\infty}$. Since $v_{Y}$ is $\left(\frac{1}{\lambda H \circ \pi}, T_{Y}\right)$-conformal, $\left.v_{Y}(\{(\underbrace{0, \ldots, 0, j, \overline{0}}_{n \text {-times }}), \pm)\}\right)=$ $v_{Y}(\{z\}) \forall n \geqslant 0$, a contradiction to the finiteness of $v_{Y}$.

Step 2. $v_{Y}\left(\left\{\left(\omega_{\beta}, \pm\right)\right\}\right)=0$. Suppose (to get a contradiction) that $v_{Y}\left(\left\{\left(\omega_{\beta}, \pm\right)\right\}\right)>0$. In this case, $\omega_{\beta} \in X_{\beta}(J)$, whence $a_{n} \in J \forall n \geqslant 1$. In particular $a_{\min }:=\min \left\{a_{n}: n \in \mathbb{N}\right\} \in J$. We construct a preimage of $\left.\left.\left(\omega_{\beta}, \pm\right)\right\}\right)$, which on one hand belongs to $Y_{\beta}(J)$ (and by $T_{Y}$-non-singularity has positive measure), and on the other hand has an infinite exchangeable orbit with infinite measure. This is a contradiction to the finiteness of $v_{Y}$.

Set $z^{ \pm}:=\left(\left(a_{\min }, \omega_{\beta}\right), \pm\right)$, then $z^{ \pm} \in Y(J)$ and its exchangeable orbit consists of $\left\{\left(a_{1}^{n-1}, a_{\min }, a_{n+1}^{\infty}\right): n \in \mathbb{N}\right\}$. This is an infinite set whenever $\omega_{\beta}$ is not eventually periodic.

Step 3. $v_{Y}\left(\pi^{-1} \Gamma\right)=0$. For every $\gamma \in \Gamma, \epsilon= \pm 1$, either $(\gamma, \epsilon) \notin Y(J)$ in which case $v_{Y}\{(\gamma, \epsilon)\}=0$, or $(\gamma, \epsilon) \epsilon$ $Y(J)$ and $\exists n, k>0$ such that $T_{Y}^{n}((\gamma, \epsilon)) \in T^{k}\left\{(\overline{0}), \omega_{\beta}\right\}$ and $\nu_{Y}\{(\gamma, \epsilon)\}=0$ by $T_{Y}$-non-singularity of $\nu_{Y}$.

As explained above, having proved that $\nu_{Y}\left(\pi^{-1} \Gamma\right)=0$, we can now obtain a non-atomic, $\left(\frac{1}{\lambda H}, T\right)$-conformal $\mu_{J, H} \in \mathcal{P}\left(X_{\beta, 0}(J)\right)$.

We turn to the uniqueness part of (1). By $\left(\frac{1}{\lambda H}, T\right)$-conformality,

$$
\mu_{J, H}(a):=\lambda^{n} \widetilde{H}(a) \text { for all } a \in \tilde{\alpha}_{k} \cap \alpha_{n}, k, n \geqslant 1
$$

where $\widetilde{H}: \bigcup_{n=1}^{\infty} \alpha_{n} \rightarrow \mathbb{R}_{+}$is defined by $\widetilde{H}\left(\left[a_{1}^{n}\right]\right):=\prod_{k=1}^{n} H\left(a_{k}\right)\left(\left[a_{1}^{n}\right] \in \tilde{\alpha} \cap \alpha_{n}\right)$. Noting that if $B_{n}:=\sum_{a \in \tilde{\alpha} \cap \alpha_{n}} \widetilde{H}(a)$, then $\sum_{n=1}^{\infty} B_{n} \lambda^{n}=1$, we see that the $\lambda>0$ appearing in (1) is unique. Eq. (8.2) thus determines $\mu_{J, H}$ uniquely. Along the way we have also shown that $\mu_{J, H}$ is a $(S, \tilde{\alpha})$-product measure.

Since $\mu_{J, H}$ is a $(S, \tilde{\alpha})$-product measure, it is $\mathcal{E}\left(X_{\beta, 0}, S, \tilde{\alpha}\right)$-invariant, and (by Theorem 2.1, part 1), $\mathcal{E}\left(X_{\beta, 0}, S, \tilde{\alpha}\right)$ ergodic, whence by $(7.3), \mathcal{E}\left(X_{\beta, 0}, T, \alpha\right)$-ergodic.

To see that it is also $\mathcal{E}\left(X_{\beta, 0}, T, \alpha\right)$-invariance, use Lemma 7.2 to observe that $\mathcal{E}\left(X_{\beta, 0}, T, \alpha\right)$ is generated by holonomies of form $(\tilde{x}, z) \mapsto(\tilde{y}, z)$ where $[\tilde{x}] \bowtie[\tilde{y}]$. These are all measure preserving.

Finally observe that since $\mu_{J, H}$ is supported on $X_{\beta, 0}$ and $X_{\beta, 0}$ is $\mathcal{E}\left(X_{\beta}, T, a\right)$ invariant, $\mathcal{E}\left(X_{\beta, 0}, T, \alpha\right)$-ergodicity and invariance is the same as $\mathcal{E}\left(X_{\beta}, T, \alpha\right)$-ergodicity and invariance.

Remark. The Lebesgue measure $m$ corresponds to $J=\{0,1, \ldots,[\beta]\}, H=$ const.

Corollary 8.1. If $X_{\beta}(J)$ is infinite, then $\mathcal{E}\left(X_{\beta, 0}, T, \alpha\right) \cap X_{\beta}(J)^{2}$ is topologically transitive.

\section{From exchangeable measures to conformal measures ( $\beta$-expansions)}

The aim of this section is to prove:

Theorem 9.0. If $v \in \mathfrak{M}\left(X_{\beta, 0}\right)$ is locally finite and $\mathcal{E}\left(X_{\beta}\right)$-invariant and ergodic, then $v=\mu_{J, H}$ for some $J \subset$ $\{0,1, \ldots,[\beta]\}, H: J \rightarrow \mathbb{R}_{+}$.

Theorem 9.0 is false if $\mathfrak{M}\left(X_{\beta, 0}\right)$ is replaced by $\mathfrak{M}\left(X_{\beta}\right)$ : If $\beta=\frac{1+\sqrt{5}}{2}, \omega_{\beta}=(\overline{1,0})$ and $\delta_{\omega_{\beta}}$ is $\mathcal{E}\left(X_{\beta}\right)$-invariant and ergodic but not of form $\mu_{J, H}$.

Lemma 9.1. Suppose that $(X, T, \alpha)$ is a fibred system s.t. $\forall x \in X, \exists N \geqslant 1$ s.t. $T^{N}\left[x_{1}, \ldots, x_{N}\right]=X$. If $\psi(x):=$ $\min \left\{N \geqslant 1: T^{N}\left[x_{1}, \ldots, x_{N}\right]=X\right\}$ and $S x:=T^{\psi(x)} x$, then $\mathfrak{T}(T)=\mathfrak{G}(S, \psi)($ see $(1.0))$. 
Proof. $\supseteq$ : Suppose that $x \stackrel{\mathfrak{G}(S, \psi)}{\sim} y$, then $\exists k, \ell \geqslant 0, S^{k} x=S^{\ell} y, \psi_{k}(x)=\psi_{\ell}(y)=: N$ whence $T^{N} x=S^{k} x=S^{\ell} y=$ $T^{N} y$ and $x \stackrel{\mathfrak{T}(T)}{\sim} y$.

$\subseteq$ : Suppose that $x \stackrel{\mathfrak{T}(T)}{\sim} y$, then $\exists N \geqslant 0, T^{N} x=T^{N} y=: z$ and $\exists \ell \geqslant 1$ so that

$\kappa:=\psi_{\ell}(z)>N+\max \left\{\psi\left(T^{j} x\right), \psi\left(T^{j} y\right): 0 \leqslant j \leqslant N\right\}$.

It suffices to show that $\kappa=\psi_{p}(x)=\psi_{q}(y)$ for some $p, q \geqslant 1$ as in this case, $S^{p} x=S^{q} y$ and $x \stackrel{\mathfrak{G}(S, \psi)}{\sim} y$.

To see that $\kappa=\psi_{p}(x)=\psi_{q}(y)$ for some $p, q \geqslant 1$ we prove that

$$
T^{N+\kappa}\left[x_{1}, \ldots, x_{N+\kappa}\right]=T^{N+\kappa}\left[y_{1}, \ldots, y_{N+\kappa}\right]=X .
$$

We will show only that $T^{N+\kappa}\left[x_{1}, \ldots, x_{N+\kappa}\right]=X$ (the other case being analogous). Suppose otherwise, and let $K:=$ $\max \left\{k \geqslant 1: T^{N+\kappa-k}\left[x_{k+1}, \ldots, x_{N+\kappa}\right] \neq X\right\}$.

By choice of $\kappa, T^{\kappa}\left[x_{N+1}, \ldots, x_{N+\kappa}\right]=X$, whence $T^{\kappa-j}\left[x_{N+j+1}, \ldots, x_{N+\kappa}\right]=X \forall 0 \leqslant j \leqslant \kappa$. It follows that $K \leqslant N-1$.

Let $L:=\psi\left(T^{K} x\right)$, then $T^{L}\left[x_{K+1}, \ldots, x_{K+L}\right]=X$. By choice of $\kappa, K+L<N+\kappa$ whence

$$
\begin{aligned}
T^{N+\kappa-K}\left[x_{K+1}, \ldots, x_{N+\kappa}\right] & =T^{N+\kappa-K}\left(\left[x_{K+1}, \ldots, x_{K+L}\right] \cap T^{-L}\left[x_{K+L+1}, \ldots, x_{N+\kappa}\right]\right) \\
& =T^{L}\left[x_{K+1}, \ldots, x_{K+L}\right] \cap T^{N+\kappa-(K+L)}\left[x_{K+L+1}, \ldots, x_{N+\kappa}\right] \\
& =T^{N+\kappa-(K+L)}\left[x_{K+L+1}, \ldots, x_{N+\kappa}\right] \neq X
\end{aligned}
$$

contradicting maximality of $K$.

Lemma 9.2. $\mathcal{E}\left(X_{\beta, 0}, T, \alpha\right)=\mathfrak{G}\left(\left.S\right|_{X_{\beta, 0}}, \Phi^{(\beta)}\right)$ where $\Phi^{(\beta)}:=\left(F^{b}, \psi\right): \quad X_{\beta, 0} \rightarrow \mathbb{G}_{\beta}:=\mathbb{Z}^{[\beta]} \times \mathbb{Z}$ and $F^{b}:=$ $\sum_{k=0}^{\psi-1} F^{\natural} \circ T^{k}$.

Proof. By Proposition 2.0, $(x, y) \in \mathcal{E}\left(X_{\beta, 0}, T, \alpha\right)$ iff $(x, y) \in \mathfrak{T}(T) \& F_{n}^{\natural}(x)=F_{n}^{\natural}(y)$ whenever $T^{n} x=T^{n} y$. By Lemma 9.1, $(x, y) \in \mathfrak{T}(T)$ iff $\exists k, \ell \geqslant 1$ with $\psi_{k}(x)=\psi_{\ell}(y)=: N$ and $S^{k} x=T^{N} x=T^{N} y=S^{k} y$. Thus $(x, y) \in$ $\mathcal{E}\left(X_{\beta, 0}, T, \alpha\right)$ iff $\exists k, \ell \geqslant 1$ such that

(1) $\psi_{k}(x)=\psi_{\ell}(y)=: N$,

(2) $S^{k} x=T^{N} x=T^{N} y=S^{k} y$, and

(3) $F_{k}^{b}(x)=F_{N}^{\natural}(x)=F_{N}^{\natural}(y)=F_{\ell}^{b}(y)$.

Equivalently $(x, y) \in \mathfrak{G}\left(S, \Phi^{(\beta)}\right)$.

Proof of Theorem 9.0. By (7.3), $v$ is $\mathcal{E}\left(X_{\beta, 0}, S, \tilde{\alpha}\right)$-invariant and $\mathfrak{T}\left(\left.S\right|_{X_{\beta, 0}}\right)$-ergodic. By Proposition $2.2, v$ is recurrent, and proportional to a $(S, \tilde{\alpha})$-product measure. In particular:

(1) for each $0 \leqslant b \leqslant[\beta], N_{b}:=\sum_{k=1}^{\infty} \delta_{x_{k}, b}=0, \infty v$-a.e.;

(2) $v$ is either a point mass or non-atomic;

(3) $v$ is $\mathfrak{G}\left(\left.S\right|_{X_{\beta, v}}\right)$-non-singular where $X_{\beta, v}:=$ the closed support of $v$ in $X_{\beta}$.

Let $J:=\left\{b \in\{0,1, \ldots,[\beta]\}: N_{b}=\infty\right\}$. Either $X_{\beta}(J)$ is a singleton and $v$ is a point mass, or $X_{\beta}(J)$ is uncountable and $\mathcal{E}\left(X_{\beta, 0}, T, \alpha\right) \cap X_{\beta}(J)^{2}$ is topologically transitive (Corollary 8.1). The first case is covered by the theorem. In the second case $X_{\beta, v}=X_{\beta}(J)$, so $v$ is not a point mass, whence by (2) non-atomic. Henceforth we restrict ourselves to this case.

Fix $j_{0}:=\min J$, and define

$$
\begin{aligned}
& F^{J, \natural}: X_{\beta}(J) \rightarrow \mathbb{Z}^{J \backslash\left\{j_{0}\right\}}, \quad \text { where } F^{J, \natural}(x)_{j}:=\delta_{x_{1}, j}, \\
& F^{J, b}: X_{\beta}(J) \rightarrow \mathbb{Z}^{J \backslash\left\{j_{0}\right\}}, \quad \text { where } F^{J, b}:=\sum_{k=0}^{\psi-1} F^{J, \natural} \circ T^{k}, \\
& \Phi=\Phi^{(J)}: X_{\beta}(J) \rightarrow \mathbb{G}, \quad \text { where } \mathbb{G}:=\mathbb{Z}^{J \backslash\left\{j_{0}\right\}} \times \mathbb{Z} \text { and } \Phi:=\left(F^{J, b}, \psi\right) .
\end{aligned}
$$


By Lemma $9.2 \mathcal{E}\left(X_{\beta}(J), T, \alpha\right)=\mathfrak{G}\left(\left.S\right|_{X_{\beta}(J)}, \Phi\right) \cong \mathfrak{G}\left(\left.S_{\Phi}\right|_{X_{\beta}(J) \times \mathbb{G}}\right) \cap\left(X_{\beta}(J) \times\{0\}\right)^{2}$. By Proposition 1.0, there is a unique $\sigma$-finite, $\mathfrak{G}\left(S_{\Phi} \mid X_{\beta}(J) \times \mathbb{G}\right)$-invariant, ergodic measure $m$ on $X_{\beta}(J) \times \mathbb{G}$ so that

$$
m(A \times\{0\})=v(A) \quad \text { for all } A \in \mathcal{B}\left(X_{\beta}(J)\right) .
$$

Step 1. $m\left(X_{\beta}(J) \times\{g\}\right)<\infty \forall g \in \mathbb{G}$. We claim first that $\nu_{g} \ll v \forall g \in \mathbb{G}$ where $\nu_{g}(A):=m(A \times\{g\})$. To see this, suppose that $B \in \mathcal{B}\left(X_{\beta}(J)\right), m(B \times\{g\})>0$, then by $\mathfrak{G}\left(\left.S_{\Phi}\right|_{X_{\beta}(J) \times \mathbb{G}}\right)$-ergodicity of $m, \exists k, \ell \geqslant 1, a \in \tilde{\alpha}_{k}, b \in \tilde{\alpha}_{\ell}$ and $A \in \mathcal{B}\left(X_{\beta}\right), v(A)>0, A \subset a$ so that $\Pi(A \times\{0\}) \subset B \times\{g\}$ where $\Pi: a \times \mathbb{G} \rightarrow b \times \mathbb{G}$ is defined by

$$
\Pi(y, z):=(\pi(y), z+\widehat{\Phi}(y, \pi(y))), \quad \pi(a, x):=(b, x) .
$$

In particular $\Pi(A) \subset B$ whence, by $\mathfrak{G}\left(\left.S\right|_{X_{\beta}(J)}\right)$-non-singularity of $v, v(B)>0$.

Next, for $g \in \mathbb{G}$, define $Q_{g}: X_{\beta}(J) \times \mathbb{G} \rightarrow X_{\beta}(J) \times \mathbb{G}$ by $Q_{g}(x, z):=(x, z+g)$. Evidently

$$
\left(Q_{g} \times Q_{g}\right)\left[\mathfrak{G}\left(\left.S_{\Phi}\right|_{X_{\beta}(J) \times \mathbb{G}}\right)\right]=\mathfrak{G}\left(\left.S_{\Phi}\right|_{X_{\beta}(J) \times \mathbb{G}}\right)
$$

whence $m \circ Q_{g}$ is also a $\sigma$-finite, $\mathfrak{G}\left(\left.S_{\Phi}\right|_{X_{\beta}(J) \times \mathbb{G}}\right)$-invariant, ergodic measure $m$ on $X_{\beta}(J) \times \mathbb{G}$. It follows that either $m \circ Q_{g} \perp m$ or $m \circ Q_{g}=c_{g} m$ for some $c_{g} \in \mathbb{R}_{+}$. In particular,

$$
m\left(X_{\beta} \times\{g\}\right)= \begin{cases}0, & m \circ Q_{g} \perp m, \\ c_{g}<\infty & \text { else. }\end{cases}
$$

Step 2. $\mathbb{H}:=\left\{h \in \mathbb{G}: m \circ Q_{h} \sim m\right\}$ is a subgroup, and $m \circ Q_{h} \perp m \forall h \notin \mathbb{H}$. We claim that it is enough to show that $\mathbb{H}=\mathbb{G}$.

Indeed, if $\mathbb{H}=\mathbb{G}$, then $m(A \times\{g\})=\mathrm{e}^{H(g)} \mu(A)$ for some homomorphism $H: \mathbb{G} \rightarrow \mathbb{R}$, whence $\frac{\mathrm{d} \mu \circ S}{\mathrm{~d} \mu}=\mathrm{e}^{-H \circ \Phi}$ and $\mu=\mu_{J, h}$ where $h:=\left.H\right|_{\mathbb{Z}^{\left.J \backslash j_{0}\right\}} \times\{0\}}$.

Step 3. $\left\langle\Phi\left(X_{\beta}(J)\right)\right\rangle=\mathbb{G}$ and $\Phi\left(X_{\beta}(J)\right) \subset \mathbb{H}$. Consequently, $\mathbb{H}=\mathbb{G}$.

Let $\left\{e_{i}\right\}$ be the standard basis for $\mathbb{Z}^{J \backslash\left\{j_{0}\right\}}$. To see the first identity, note that for every $j \in J, j \neq[\beta],[j] \in \tilde{\alpha}$ and so $\left(e_{j}, 1\right)=\Phi([j]) \in \Phi\left(X_{\beta}(J)\right)\left(j \in J \backslash\left\{j_{0},[\beta]\right\}\right)$ and $(0,1)=\Phi\left(\left[j_{0}\right]\right) \in \Phi\left(X_{\beta}(J)\right)$.

In case $[\beta] \in J, \#\left\{n \geqslant 1: \omega_{n}=[\beta]\right\}=\infty$ and $\exists N \geqslant 1$ with $\omega_{1}^{N}=\left([\beta], \omega_{2}, \ldots, \omega_{N-1},[\beta]\right)$ where $\omega_{2}, \ldots, \omega_{N-1} \neq$ $[\beta]$, whence $\left[[\beta], j_{0}^{k}\right] \in \tilde{\alpha}$ for some $1 \leqslant k \leqslant N-1$ and $\left(e_{[\beta]}, k+1\right)=\Phi\left(\left[[\beta], j_{0}^{k}\right]\right) \in \Phi\left(X_{\beta}(J)\right)$.

Next, we show that $\Phi\left(X_{\beta}(J)\right) \subset \mathbb{H}$. Accordingly, let $\Phi \equiv h$ on $a \in \tilde{\alpha}$, and assume by way of contradiction that $h \notin \mathbb{H}$. In this case, $m \circ Q_{h} \perp m$ and $\exists Z \in \mathcal{B}(X), m(Z \times\{0\})=1, m(Z \times\{-h\})=0$. It follows that $\exists \xi \subset \bigcup_{n=1}^{\infty} \tilde{\alpha}_{n}$ countable, such that $U:=\bigcup_{A \in \xi} A \supset Z$ and $m(U \times\{-h\})<\frac{1}{3}$.

Since $m(U \times\{0\})=1, \exists A \in \xi$ such that $m(A \times\{-h\})<\frac{1}{3} m(A \times\{0\})$.

Define $\kappa: A \rightarrow A$ by $\kappa(A, x):=(A, a, x)$. Evidently $(x, \kappa(x)) \in \mathfrak{G}(S) \forall x \in A$, and $\widetilde{\Phi}(x, \kappa(x))=-h \forall x \in A$. Thus, if $\tilde{\kappa}: A \times \mathbb{G} \rightarrow A \times \mathbb{G}$ is defined by $\tilde{\kappa}(x, g):=(\kappa(x), g-h)$, then $((x, g), \tilde{\kappa}(x, g)) \in \mathfrak{G}\left(S_{\Phi}\right) \forall(x, g) \in A \times \mathbb{G}$. It follows that

$$
m(A \times\{0\})=m(\tilde{\kappa}(A \times\{0\})) \leqslant m(A \times\{-h\})<\frac{1}{3} m(A \times\{0\}) .
$$

By the contradiction, $h \in \mathbb{H}$, proving the step and with it the theorem.

\section{References}

[1] J. Aaronson, M. Denker, Group extensions of Gibbs-Markov maps, Probab. Theory Related Fields 123 (1) (2002) 38-40.

[2] J. Aaronson, M. Denker, M. Urbański, Ergodic theory for Markov fibred systems and parabolic rational maps, Trans. Amer. Math. Soc. 337 (2) (1993) 495-548.

[3] J. Aaronson, M. Denker, O. Sarig, R. Zweimüller, Aperiodicity of cocycles and conditional local limit theorems, Stoch. Dyn. 4 (1) (2004) $31-62$.

[4] J. Aaronson, H. Nakada, O. Sarig, R. Solomyak, Invariant measures and asymptotics for some skew products, Israel J. Math. 128 (2002) 93-134.

[5] J. Aaronson, H. Nakada, O. Sarig, R. Solomyak, Corrections to: Invariant measures and asymptotics for some skew products, Israel J. Math. 138 (2003) 377-379.

[6] F. Blanchard, $\beta$-expansions and symbolic dynamics, Theoret. Comput. Sci. 65 (2) (1989) 131-141.

[7] K.L. Chung, Markov Chains with Stationary Transition Probabilities, Springer, Heidelberg, 1960.

[8] P. Diaconis, D. Freedman, De Finetti's theorem for Markov chains, Ann. Probab. 8 (8) (1980) 115-130.

[9] E. Effros, Transformation groups and $C^{*}$-algebras, Ann. of Math. (2) 81 (1965) 38-55. 
[10] J. Feldman, C.C. Moore, Ergodic equivalence relations, cohomology, and von Neumann algebras. I, Trans. Amer. Math. Soc. 234 (2) (1977) 289-324.

[11] L. Fuchs, Abelian Groups, Internat. Ser. Monogr. Pure Appl. Math., Pergamon Press, New York, 1960.

[12] A.O. Gel'fond, A common property of number systems, Izv. Akad. Nauk SSSR. Ser. Mat. 23 (1959) 809-814 (in Russian).

[13] J. Glimm, Locally compact transformation groups, Trans. Amer. Math. Soc. 101 (1961) 124-138.

[14] G. Greschonig, K. Schmidt, Ergodic decomposition of quasi-invariant probability measures, part 2, Colloq. Math. 84/85 (2000) $495-514$.

[15] L.A. Grigorenko, On the $\sigma$-algebra of symmetric events for a countable Markov chain, Theory Probab. Appl. 24 (1979) $199-204$.

[16] E. Hewitt, L.J. Savage, Symmetric measures on Cartesian products, Trans. Amer. Math. Soc. 80 (1955) 470-501.

[17] S. Ito, Y. Takahashi, Markov subshifts and realization of $\beta$-expansions, J. Math. Soc. Japan 26 (1974) 33-55.

[18] A.N. Livšic, Certain properties of the homology of $U$-systems, Mat. Zametki 10 (1971) 555-564. English Transl. in Math. Notes 10 (1971) 758-763.

[19] D. Maharam, Incompressible transformations, Fund. Math. 56 (1964) 35-50.

[20] P.-A. Meyer, Probability and Potentials, Blaisdell Publishing Co, Ginn and Co, Waltham, MA, 1966.

[21] W. Parry, On the $\beta$-expansions of real numbers, Acta Math. Acad. Sci. Hungar. 11 (1960) 401-416.

[22] W. Parry, K. Schmidt, Natural coefficients and invariants for Markov-shifts, Invent. Math. 76 (1) (1984) 15-32.

[23] K. Petersen, K. Schmidt, Symmetric Gibbs measures, Trans. Amer. Math. Soc. 349 (1997) 2775-2811.

[24] A. Rényi, Representations for real numbers and their ergodic properties, Acta Math. Acad. Sci. Hungar. 8 (1957) $477-493$.

[25] O. Sarig, Existence of Gibbs measures for countable Markov shifts, Proc. Amer. Math. Soc. 131 (6) (2003) 1751-1758.

[26] K. Schmidt, Infinite invariant measures on the circle, in: Convegno sulle Misure su Gruppi e su Spazi Vettoriali, Convegno sui Gruppi e Anelli Ordinati, INDAM, Rome, 1975, in: Symposia Mathematica, vol. XXI, Academic Press, London, 1977, pp. $37-43$.

[27] F. Schweiger, Ergodic Theory of Fibred Systems and Metric Number Theory, Oxford Science Publications, The Clarendon Press, Oxford University Press, New York, 1995.

[28] M. Smorodinsky, $\beta$-automorphisms are Bernoulli shifts, Acta. Math. Acad. Sci. Hungar. 24 (1973) 273-278.

[29] D. Vere-Jones, Ergodic properties of nonnegative matrices. I, Pacific J. Math. 22 (1967) 361-386.

[30] P. Walters, Equilibrium states for $\beta$-transformations and related transformations, Math. Z. 159 (1) (1978) 65-88. 